\title{
Clavibacter michiganensis subsp. michiganensis Vatr1 and Vatr2 Transcriptional Regulators Are Required for Virulence in Tomato
}

\author{
Alon Savidor, ${ }^{1,2}$ Laura Chalupowicz, ${ }^{3}$ Doron Teper, ${ }^{1}$ Karl-Heinz Gartemann,, ${ }^{1,4}$ Rudolf Eichenlaub, ${ }^{4}$ \\ Shulamit Manulis-Sasson, ${ }^{3}$ Isaac Barash, ${ }^{1}$ and Guido Sessa ${ }^{1}$ \\ ${ }^{1}$ Department of Molecular Biology and Ecology of Plants, Tel Aviv University, Tel Aviv 69978, Israel; ${ }^{2}$ The Nancy \& Stephen \\ Grand Israel National Center for Personalized Medicine, Weizmann Institute of Science, Rehovot 7610001, Israel; ${ }^{3}$ Department \\ of Plant Pathology and Weed Research, ARO, the Volcani Center, Bet Dagan 50250, Israel; ${ }^{4}$ Department of Genetechnology/ \\ Microbiology, Faculty of Biology, University of Bielefeld, 33501 Bielefeld, Germany
}

Submitted 27 February 2014. Accepted 9 June 2014.

\begin{abstract}
The plant pathogen Clavibacter michiganensis subsp. michiganensis is a gram-positive bacterium responsible for wilt and canker disease of tomato. Although disease development is well characterized and diagnosed, molecular mechanisms of $C$. michiganensis subsp. michiganensis virulence are poorly understood. Here, we identified and characterized two $C$. michiganensis subsp. michiganensis transcriptional regulators, Vatr1 and Vatr2, that are involved in pathogenicity of $C$. michiganensis subsp. michiganensis. Vatr1 and Vatr2 belong to TetR and MocR families of transcriptional regulators, respectively. Mutations in their corresponding genes caused attenuated virulence, with the $\Delta v a t r 2$ mutant showing a more dramatic effect than $\Delta v a t r 1$. Although both mutants grew well in vitro and reached a high titer in planta, they caused reduced wilting and canker development in infected plants compared with the wild-type bacterium. They also led to a reduced expression of the ethylene-synthesizing tomato enzyme ACC-oxidase compared with wild-type $C$. michiganensis subsp. michiganensis and to reduced ethylene production in the plant. Transcriptomic analysis of wild-type $C$. michiganensis subsp. michiganensis and the two mutants under infectionmimicking conditions revealed that Vatr1 and Vatr2 regulate expression of virulence factors, membrane and secreted proteins, and signal-transducing proteins. A $70 \%$ overlap between the sets of genes positively regulated by Vatr1 and Vatr2 suggests that these transcriptional regulators are on the same molecular pathway responsible for $C$. michiganensis subsp. michiganensis virulence.
\end{abstract}

The gram-positive bacterium Clavibacter michiganensis subsp. michiganensis is the causal agent of wilt and canker disease of tomato (Solanum lycopersicum), one of the most important diseases of tomato crops worldwide (Davis et al. 1984). The bacterium invades the plant through wounds or natural openings and then colonizes the xylem vessels in the

Corresponding author: Guido Sessa; Telephone: +972.3.640.9766; Fax: +972.3.640.9380; E-mail: guidos@post.tau.ac.il

* The $\boldsymbol{e}$-Xtra logo stands for "electronic extra" and indicates that six supplementary figures and a supplementary table are published online.

(c) 2014 The American Phytopathological Society plant stem, where it can reach a very high titer $\left(>10^{9} \mathrm{CFU} / \mathrm{g}\right.$ of tissue) within a few days (Meletzus et al. 1993). Because xylem vessels span the entire plant, the bacterium can spread to the whole plant and the disease turns systemic. Disease symptoms include leaf wilting and canker on the stem, which may lead to plant death. C. michiganensis subsp. michiganensis can also colonize the fruit and seed, and infected seed are a major source of disease spreading (Fatmi et al. 1991; Tsiantos 1987). Although several wild tomato species have been shown to be resistant to $C$. michiganensis subsp. michiganensis (Coaker et al. 2004), there are no commercial tomato cultivars that are resistant to $C$. michiganensis subsp. michiganensis infection.

The wild-type strain $C$. michiganensis subsp. michiganensis NCPPB382 (hereafter referred to as Cmm382) carries two plasmids, pCM1 and pCM2 (Meletzus and Eichenlaub 1991), both of which are required for full virulence because they encode the virulence factors CelA (Jahr et al. 2000) and Pat-1 (Dreier et al. 1997), respectively. Homologs of the Pat-1 serine protease are found on pCM2 as well as on the chromosome, and represent one out of three families of extracellular serine proteases identified in the $C$. michiganensis subsp. michiganensis genome (Gartemann et al. 2008). A second family is the chymotrypsin-related Ppa family of serine proteases, which includes 11 members, and the third is the 3-member family of subtilase proteases. Among these serine proteases, ChpC, $\mathrm{PpaA}$, and PpaC were shown to have an effect on $C$. michiganensis subsp. michiganensis virulence or colonization-ability (Gartemann et al. 2008). Another genomic region important for C. michiganensis subsp. michiganensis virulence is the pathogenicity island, which is located in the chromosome and encodes proteins important for plant colonization (Gartemann et al. 2008). Although a few C. michiganensis subsp. michiganensis proteins that affect virulence and colonization have been identified, the molecular pathways leading to pathogenicity are unknown.

Ethylene has been shown to play a major role in disease symptom development, as is evident from the significantly delayed leaf wilting in tomato plants that are impaired in ethylene perception or production (Balaji et al. 2008, 2011). In a previous proteomic study, we showed that $C$. michiganensis subsp. michiganensis specifically triggers activation of the host ACC-oxidase (ACO), the last enzyme in the ethylene biosynthesis pathway, leading to increased ethylene production and symptom development (Savidor et al. 2012). The same study showed that, upon $C$. michiganensis subsp. michiganensis infec- 
tion, tomato plants activate basal defense responses, although partial, with some suppressed components. C. michiganensis subsp. michiganensis, on the other hand, senses the plant environment and transduces signals that result in elevated expression and secretion of hydrolytic enzymes whose substrates are likely host molecules. Several putative $C$. michiganensis subsp. michiganensis signal-transducing proteins were upregulated during infection and were identified as candidates for involvement in C. michiganensis subsp. michiganensis virulence. Here, we describe characterization of two of them, CMM_2645 and CMM_2969, which we have named virulence-associated transcriptional regulator (Vatr)1 and Vatr2, respectively. We show that these proteins regulate $C$. michiganensis subsp. michiganensis virulence during infection, and that inactivation of their respective genes leads to reduced ethylene synthesis and dramatic reduction in disease symptom development in infected plants. Vatr1 and Vatr2 transcriptionally regulate virulence factors and membrane and secreted proteins, along with other transcriptional regulators.

\section{RESULTS}

Mutations in the vatr1 and vatr2 genes cause reduced disease symptoms.

Based on the results from our previously performed proteomic study (Savidor et al. 2012), we identified several candidate C. michiganensis subsp. michiganensis proteins, which may participate in virulence-specific signal transduction. Two of them, CMM_2645 and CMM_2969 (Vatr1 and Vatr2, respectively), were selected for in-depth functional analysis due to their putative function and structure: both proteins are putative transcriptional regulators containing DNA binding motifs, where Vatr1 is a protein belonging to the TetR family of transcriptional repressors and Vatr2 is a member of the GntR family of transcriptional regulators (Supplementary Fig. S1). Targeted mutagenesis of the vatrl and vatr 2 genes was carried out by insertion of a chloramphenicol cassette into their coding regions. Southern blotting with labeled probes specific for the coding sequences vatrl or vatr2 against NcoI-digested total DNA of wild-type Cmm382 and the mutants produced the expected bands: for the $\Delta v a t r 1$ mutant, bands appeared at 2.5 and $4.8 \mathrm{~kb}$ instead of 2.0 and $4.0 \mathrm{~kb}$ for the wild type (Supplementary Fig. S2). For $\Delta v a t r 2$, bands appeared at 1, 4.4, and $7.9 \mathrm{~kb}$ instead of 4.0 and $7.9 \mathrm{~kb}$ for the wild type. These results confirmed that a single insertion event of the cassette into the expected sites in the genomes of the mutants occurred and that no functional copy of the respective genes remained. Polymerase chain reaction (PCR) analysis of the pCM2 encoded pat-1 gene demonstrated that both mutants maintained the native pCM2 plasmid, which is sometimes lost during transformation (data not shown).

To test virulence of the mutant $C$. michiganensis subsp. michiganensis strains, tomato plants were inoculated with wildtype $C m m 382, \Delta v a t r 1$, or $\Delta v a t r 2$ bacterial strains and moni-

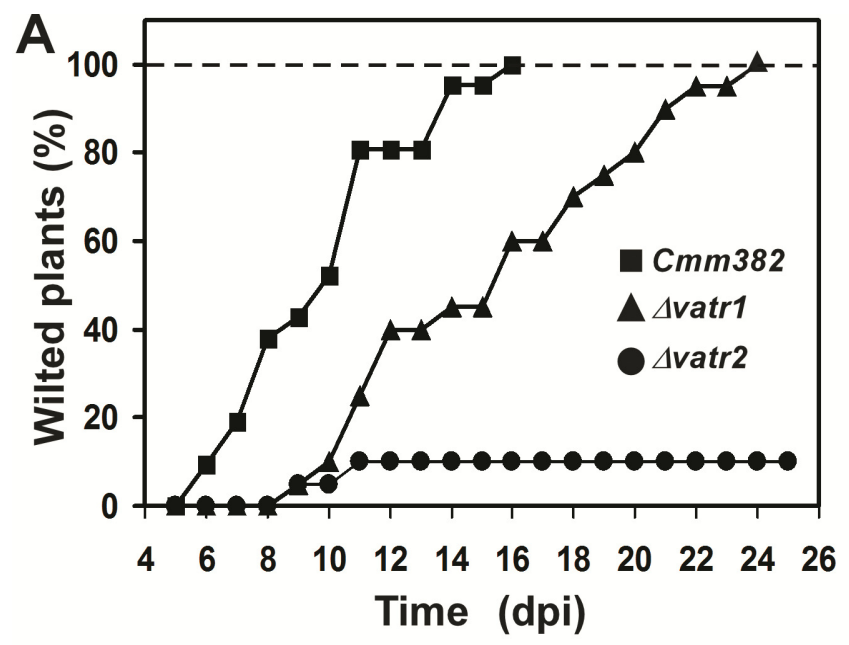

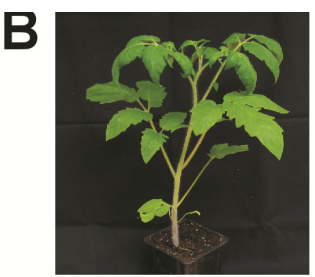

Mock

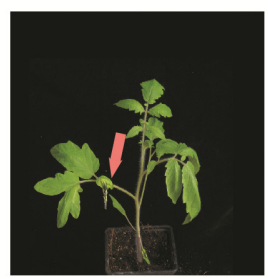

$\Delta v a t r 1$

D
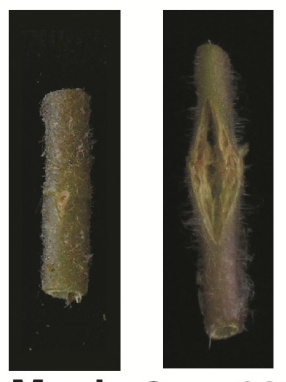

Mock Cmm382 dvatr1
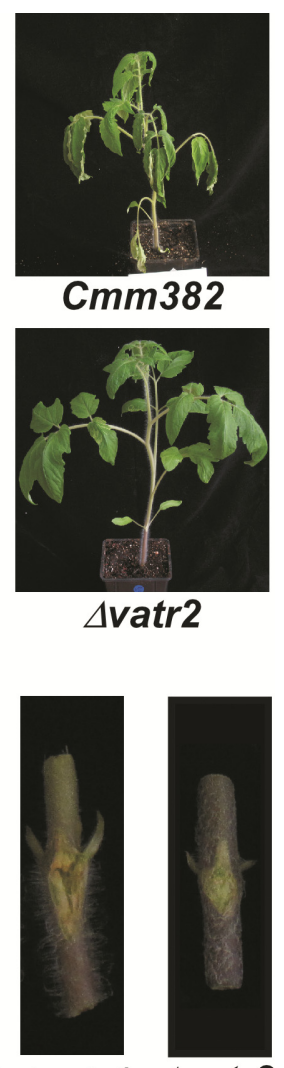

$\Delta v a t r 2$

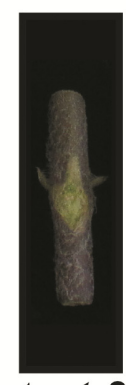

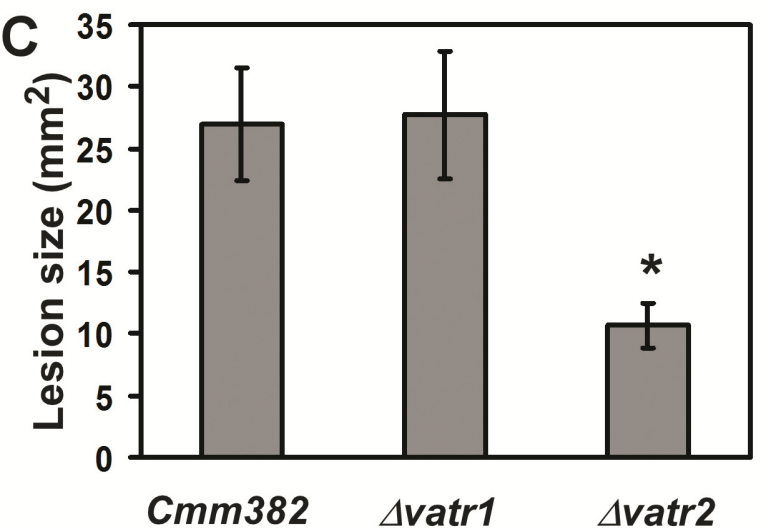

$\Delta$ vatr2

Fig. 1. Mutants $\Delta v a t r 1$ and $\Delta v a t r 2$ cause reduced disease symptoms in infected plants. Five-week-old tomato plants were inoculated with wild-type Cmm382, $\Delta$ vatr1, or $\Delta v a t r 2$. A, Plants were monitored for wilting of the first leaf at different days postinoculation (dpi). For plants infected with wild-type Cmm382 and $\Delta v a t r 1$, the experiment was stopped when plants reached $100 \%$ wilting (dotted line). B, Typical wilting in infected tomato plants at 10 dpi. The red arrow in the $\Delta$ vatrl panel points to a wilted leaf among others that had not yet wilted. $\mathbf{C}$, Canker size of infected plants at 15 dpi. Bars represent average and standard deviation $(n=15)$. An asterisk $(*)$ represents a statistically significant difference between the mutant and the wild type according to a $t$ test with $P$ value $<0.05$. D, Typical canker in infected tomato stems at $15 \mathrm{dpi}$. Experiments were repeated three times with similar results. 
tored for development of disease symptoms. Plants infected with either of the mutants showed a significant reduction in wilting compared with plants infected with wild-type $C m m 382$ (Fig. 1A and B). The wilting phenotype of $\Delta v a t r 2$-infected plants was much more dramatic than that of $\Delta v a t r l$-infected plants, and $\Delta v a t r 2$-infected plants also showed a significant reduction in stem canker size (Fig. 1C and D). To confirm that the mutant genes are the ones responsible for the reduced symptom development, a complementation assay was carried out (Fig. 2). The complementation cloning vector pHN216 is based on the native pCM2 plasmid and contains its origin of replication (Laine et al. 1996). When transformed with this vector, C. michiganensis subsp. michiganensis strains lose their native pCM2 plasmid under antibiotic selection and become less virulent. The loss of pCM2 was shown for both complemented mutants via PCR using the pat-1 diagnostic primers (data not shown). Therefore, in order to have a correct reference for the complementation assay, both mutants were cured of pCM2. Cmm101, which is wild-type Cmm382 strain cured of pCM2, was also used as a reference. The virulence of the complemented mutants was restored (Fig. 2), confirming that the inactivation of their respective genes was responsible for the attenuated virulence.

Growth of $\Delta v a t r 1$ and $\Delta v a t r 2$ mutants in vitro and in planta.

To exclude the possibility that Vatr1 and Vatr 2 are involved in house-keeping processes that are essential for normal growth and development of the bacterium, the $\Delta v a t r l$ and $\Delta$ vatr 2 mutants, as well as wild-type $C m m 382$, were grown in vitro in rich medium (Luria-Bertani $[\mathrm{LB}]$ ) at $28^{\circ} \mathrm{C}$ and monitored for growth. Both mutants grew at the same rate as the wild type (Fig. 3A), indicating that Vatr1 and Vatr2 are not involved in housekeeping functions. We previously showed that tomato-sap-supplemented M9 minimal medium (infectionmimicking medium [IMM]) is a very good mimic of in planta conditions and that $C$. michiganensis subsp. michiganensis behaves similarly to in-planta-grown bacteria under these conditions (Savidor et al. 2012). Interestingly, when grown in IMM,

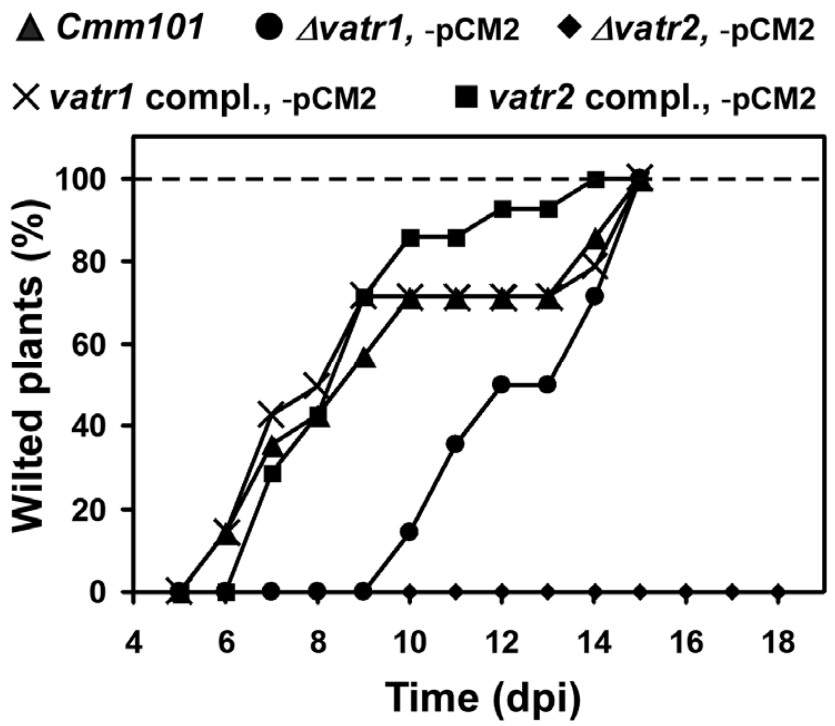

Fig. 2. Complementation of vatr1 and vatr2 in their respective mutants restores virulence. Twenty-four 5-week-old tomato plants were inoculated with Cmm101 wild-type (cured of pCM2), $\Delta$ vatr1 (cured of pCM2), $\Delta$ vatr2 (cured of pCM2), vatr1 complemented strain (cured of $\mathrm{pCM} 2$ ), or vatr 2 complemented strain (cured of $\mathrm{pCM} 2$ ) and were monitored for wilting of the first leaf at different days postinoculation (dpi). The experiment was stopped when plants reached $100 \%$ wilting (dotted line). The experiment was repeated three times with similar results. the $\Delta$ vatr 2 mutant showed attenuated growth compared with Cmm382, whereas $\Delta$ vatrl growth was comparable with Cmm382 (Fig. 3B). Thus, vatr2 may also be involved in metabolic pathways that are activated during infection, or may be affected by tomato components in the sap.
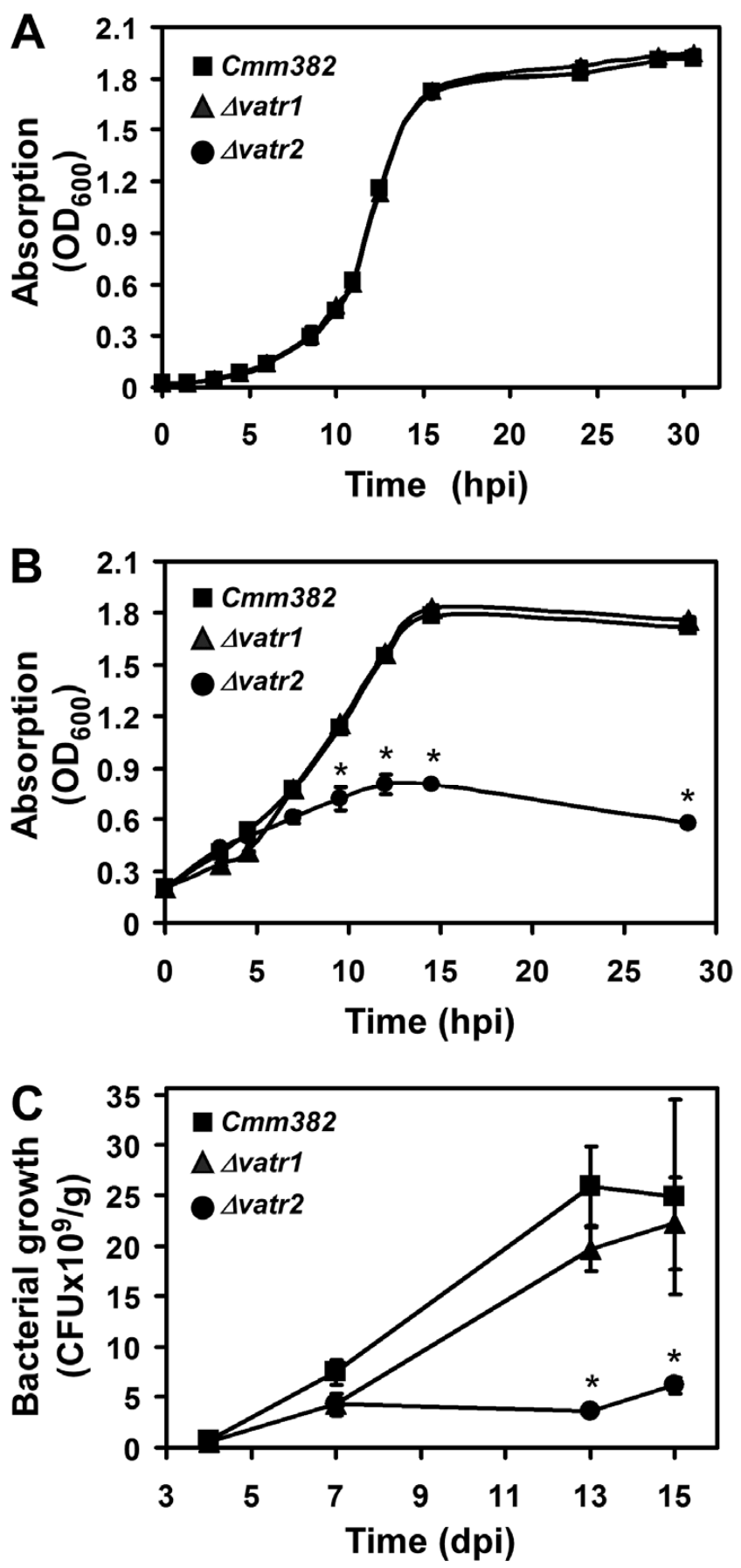

Fig. 3. In vitro and in planta growth of $\Delta v a t r 1$ and $\Delta v a t r 2$ mutants. Twenty milliliters of A, Luria-Bertani (LB) medium or $\mathbf{B}$, infection-mimicking medium were inoculated with $\mathrm{Cmm} 382$, $\Delta v a t r 1$, or $\Delta v a t r 2$ to an initial optical density at $600 \mathrm{~nm}\left(\mathrm{OD}_{600}\right)$ of 0.02 or 0.2 , respectively, and culture density was monitored for $30 \mathrm{~h}$ postinoculation (hpi). Error bars represent the standard error $(n=3)$. C, Five-week-old tomato plants were inoculated with Cmm382, svatr1, or $\Delta v a t r 2$. At 4, 7, 13, and 15 days postinoculation (dpi), a $0.5-\mathrm{cm}$ stem section was cut at $1 \mathrm{~cm}$ above the inoculation site. Stem sections were weighted, homogenized, serially diluted, and plated on LB agar, and CFU were calculated. Error bars represent the standard error $(n=3)$. Asterisks (*) represent statistically significant differences between mutants and wild type at the indicated time points according to a $t$ test with $P$ value $<$ 0.05 . Experiments were repeated three times with similar results. 
To test the ability of $\Delta v a t r l$ and $\Delta v a t r 2$ to grow in planta, tomato plants were infected with the mutants or with wild-type Cmm382, and bacterial titer was monitored at 4, 7, 13, and 15 days postinoculation (Fig. 3C). Although the $\Delta$ vatrl mutant showed similar bacterial titer over the course of infection as $C m m 382$, the titer of $\Delta v a t r 2$ was reduced approximately fivefold compared with $C m m 382$. Still, even this reduction in the ability of $\Delta v a t r 2$ to grow in planta is not sufficient by itself to explain the dramatic attenuation in virulence of the mutant, because $\Delta$ vatr 2 still reached a very high bacterial titer $\left(5 \times 10^{9}\right.$ CFU/g of tissue), which typically leads to significant symptom development in plants infected with the wild type Cmm382 (Meletzus et al. 1993; Stork et al. 2008) (Fig. 1).

\section{Mutants $\Delta v a t r 1$ and $\Delta v a t r 2$ reduce ethylene production in infected plants.}

The plant hormone ethylene has previously been shown to be an important factor in symptom development of $\mathrm{Cmm382-}$ infected tomato plants (Balaji et al. 2008). To test whether $\Delta v a t r l$ and $\Delta$ vatr 2 cause reduced ethylene production in infected plants, tomato plants were inoculated with wild-type Cmm382 or the mutants, or were mock inoculated. Disease was allowed to develop up to 15 days postinoculation, the time
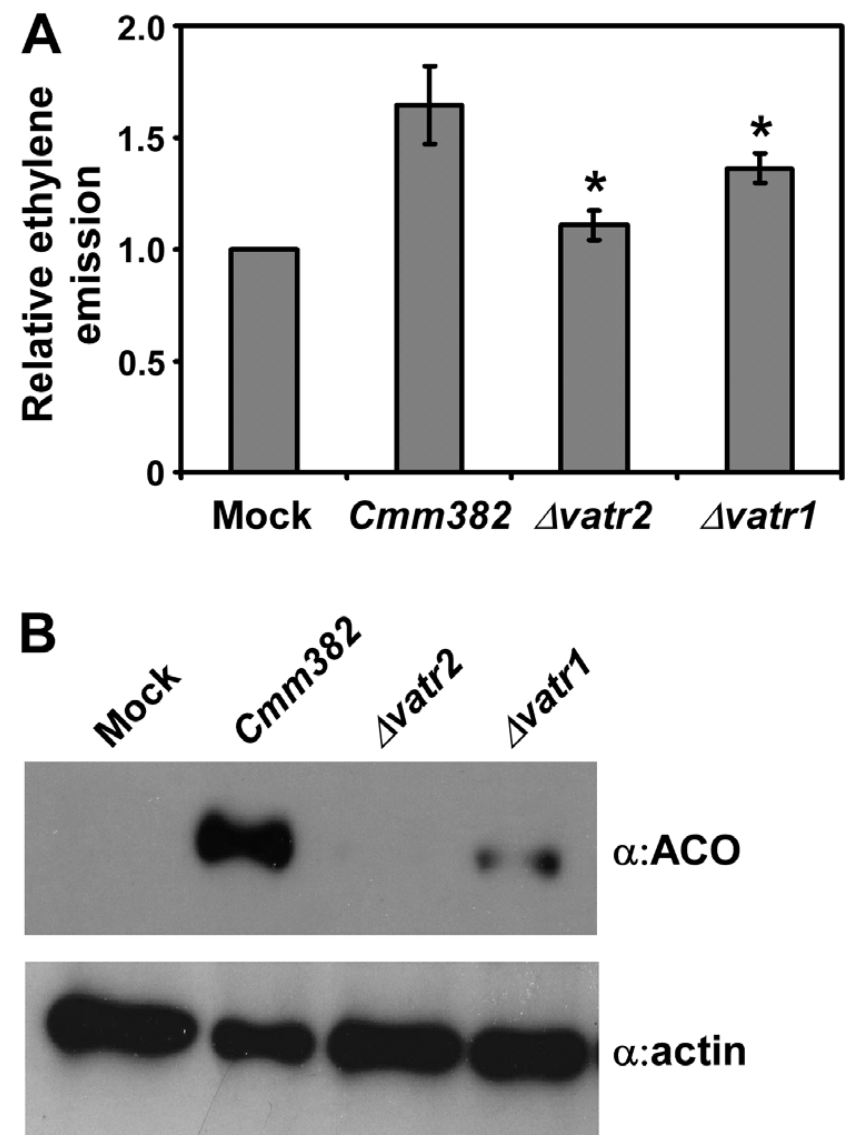

Fig. 4. Mutants $\Delta v a t r 1$ and $\Delta v a t r 2$ cause reduced ethylene emission from inoculated tomato stems. Five-week-old tomato plants were inoculated with wild-type Cmm382, svatrl, $\Delta$ vatr2, or sterile water (mock-inoculated). A, Ethylene emission from stems of infected plants measured at 15 days postinoculation (dpi) by gas chromatography. Three biological replicates were performed and the ratio of emitted ethylene between plants infected with wild-type or mutant bacteria and mock-inoculated plants was calculated. Asterisks (*) represent statistically significant differences between mutants and wild type according to a $t$ test with $P$ value $<0.05$. B , Western blot analysis of total proteins that were extracted from stems at 8 dpi, using antibodies raised against ACC-oxidase ( $\alpha$ :ACO) or actin ( $\alpha$ :actin), as reference. point in which ethylene levels in Cmm382-infected plants reach the maximum (Balaji et al. 2008), and ethylene emission from stem sections of infected plants was measured. Plants infected with either of the mutants produced reduced levels of ethylene compared with the Cmm382-infected plants (Fig. 4A). The $\Delta v a t r 2$-infected plants showed the most dramatic reduction in ethylene level, reaching only slightly above ethylene levels of mock-inoculated plants, whereas $\Delta$ vatrl-infected plants showed a more moderate reduction.

We previously showed that, in infected plants, Cmm382 specifically upregulates the tomato enzyme ACO, which catalyzes the last step in ethylene biosynthesis in the plant (Savidor et al. 2012). This upregulation is correlated with ethylene levels and development of disease symptoms. To test whether the reduced ethylene production in the mutant-inoculated plants is correlated with ACO levels, Western blotting of protein extracts from stem pieces of inoculated plants was performed using $\alpha: A C O$ antibodies. Similar to ethylene levels, ACO levels of $\Delta$ vatr2-inoculated plants were drastically below those of Cmm382-inoculated plants, whereas $\Delta v a t r l$-inoculated plants showed a more moderate reduction (Fig. 4B).

\section{Bioinformatic analysis of Vatr1 and Vatr2.}

Vatr1 is a protein of 207 amino acids $(22.5 \mathrm{kDa})$ belonging to the TetR family of transcriptional regulators. Proteins in this family are transcriptional repressors that control genes whose products are involved in different cellular processes, including different catabolic pathways, antibiotics biosynthesis, and pathogenicity (Ramos et al. 2005). Like other members of the TetR family, Vatr1 is predicted to contain an N-terminal helixturn-helix (HTH) domain (residues 22 to 61), which is typically thought to be responsible for the specific binding to DNA and facilitates regulation of its target genes (e.g., by recruiting or preventing binding of RNA-polymerase to promoter regions). No other known domains could be deduced from the Vatr1 amino acid sequence. Bacterial transcriptional regulators often control the expression of genes located in their proximity in the genome. The genes adjacent to vatrl in the $C$. michiganensis

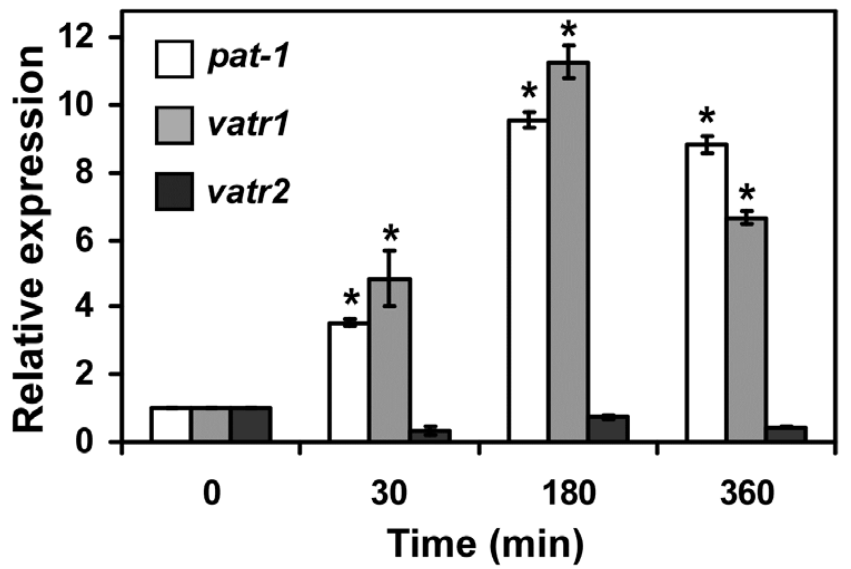

Fig. 5. Timeline transcriptional expression of the pat-1, vatrl, and vatr2 genes in infection-mimicking medium (IMM). Cmm382 bacteria were grown in Luria-Bertani medium to midexponential phase and were transferred to IMM. Samples were harvested at different time points after transfer to IMM. RNA was extracted from the harvested cells and was subjected to quantitative polymerase chain reaction with pat-1, vatr1, and vatr2 gene-specific primers, using gyrA as the normalizer. For each gene, expression at the different time points is shown relative to that at the time of transfer (time 0). Error bars represent standard error of triplicate repeats. The experiment was repeated three times with similar results. Asterisks $(*)$ represent statistically significant differences in the expression of the examined genes at the indicated time points compared with $0 \mathrm{~h}$ postinoculation, according to a $t$ test with $P$ value $<0.05$. 
subsp. michiganensis genome encode two DNA helicases, a phospholipase, a NUDIX hydrolase, and a hypothetical protein. Thus, a direct link to virulence is not immediately evident from the location of vatrl in the genome.
Vatr2 is a putative 440-residue-long protein belonging to the GntR family of transcriptional regulators. GntR proteins are either repressors or activators of transcription, regulating a wide variety of biological processes (Rigali et al. 2002). This

Table 1. List of genes positively regulated by Vatr1 or Vatr2 in infection-mimicking conditions ${ }^{\mathrm{a}}$

\begin{tabular}{|c|c|c|c|c|c|c|c|}
\hline Category, locus & Annotation & $\begin{array}{c}\mathrm{Cmm382} \\
\left(_{\text {IMM/LB })^{\mathrm{b}}}\right.\end{array}$ & $\begin{array}{c}\Delta v a t r 1 \\
(\mathbf{I M M} / \mathbf{L B})^{\mathrm{b}}\end{array}$ & $\begin{array}{c}\Delta v a t r 2 \\
(\mathbf{I M M} / \mathbf{L B})^{\mathbf{b}}\end{array}$ & 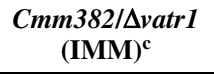 & 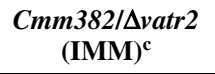 & $\underset{\text { by }}{\text { Regulated }}$ \\
\hline \multicolumn{8}{|l|}{ Virulence factors } \\
\hline \multicolumn{8}{|l|}{ CMM_0328- } \\
\hline CMM__0331 & Yersiniabactin synthesis operon & $* 21.8$ & 1.4 & 0.6 & $* * 2.8$ & $* * 4.2$ & Vatr1/2 \\
\hline CMM_2536 & SbtC serine protease & *7.4 & $* 2.5$ & $* 4.0$ & $* * 2.6$ & 1.1 & Vatr1 \\
\hline pCM2_0053 & PhpA extracellular serine protease & $* 5.4$ & 2.2 & 1.6 & $* * 1.9$ & $* * 2.5$ & Vatr2 \\
\hline pCM2_0054 & Pat-1 extracellular serine protease & $* 4.3$ & 2.0 & 1.8 & $* * 2.0$ & $* * 2.2$ & Vatr1/2 \\
\hline pCM1_0020 & CelA extracellular cellulase & $* 3.2$ & 1.0 & 1.1 & $* * 2.5$ & 1.5 & Vatr1 \\
\hline \multicolumn{8}{|c|}{ Signal transduction } \\
\hline CMM_0118 & TetR-family transcriptional regulator & $* 2.9$ & 1.2 & 1.3 & $* * 2.2$ & $* * 2.3$ & Vatr1/2 \\
\hline CMM_0119 & TetR-family transcriptional regulator & $* 3.7$ & 1.5 & 1.7 & $* * 2.4$ & 2.2 & Vatr1 \\
\hline CMM_0749 & $\begin{array}{l}\text { MarR-family transcriptional } \\
\text { regulator }\end{array}$ & $* 3.6$ & 1.7 & 1.3 & $* * 3.0$ & 2.0 & Vatr1 \\
\hline $\begin{array}{l}\text { CMM_2353, } \\
\text { CMM_2354 }\end{array}$ & $\begin{array}{l}\text { Transcriptional regulator, membrane } \\
\text { protein }\end{array}$ & $* 5.4$ & 1.8 & 0.8 & $* * 3.3$ & $* * 4.4$ & Vatr1/2 \\
\hline CMM_2683 & $\begin{array}{l}\text { Bifunctional phosphatase/glycosyl } \\
\text { transferase }\end{array}$ & $* 3.5$ & 0.6 & 0.2 & $* * 3.0$ & $* * 6.8$ & Vatr1/2 \\
\hline CMM_2849 & Phosphatase & $* 4.2$ & 1.2 & 0.8 & 1.7 & $* * 3.0$ & Vatr2 \\
\hline \multicolumn{8}{|l|}{ Chaperones } \\
\hline CMM_0316 & ClpY chaperone & $* 4.1$ & 1.6 & 1.0 & $* * 1.8$ & $* * 2.2$ & Vatr2 \\
\hline CMM_2092 & HSP20 heat shock protein & $* 4.2$ & 1.1 & 1.2 & $* * 4.0$ & $* * 2.8$ & Vatr1/2 \\
\hline CMM_2478 & GroEL HSP60 kDa chaperonin & $* 4.2$ & 1.2 & 1.8 & $* * 3.1$ & $* * 2.2$ & Vatr $1 / 2$ \\
\hline \multicolumn{8}{|c|}{ Membrane proteins } \\
\hline CMM_0117 & MFS-type efflux protein & $* 2.9$ & 1.4 & 0.4 & $* * 2.0$ & $* * 4.1$ & Vatr1/2 \\
\hline CMM_0208 & $\begin{array}{l}\text { Membrane-associated phospholipid } \\
\text { phosphatase }\end{array}$ & $* 4.1$ & 1.1 & 0.7 & $* * 3.4$ & $* * 3.4$ & Vatr1/2 \\
\hline CMM_0209 & $\begin{array}{l}\text { Membrane protein, unknown } \\
\text { function }\end{array}$ & $* 2.2$ & 0.9 & 0.6 & 1.5 & $* * 2.6$ & Vatr2 \\
\hline CMM_0285 & DMT family permease & $* 6.7$ & 1.1 & 1.0 & $* * 2.2$ & 2.0 & Vatr1 \\
\hline CMM_1226 & $\begin{array}{l}\text { Membrane protein, unknown } \\
\text { function }\end{array}$ & $* 7.6$ & $* 3.8$ & 0.8 & 2.0 & $* * 3.8$ & Vatr2 \\
\hline CMM_1524 & $\begin{array}{l}\text { Nicotinamide mononucleotide } \\
\text { transporter }\end{array}$ & $* 6.5$ & $* 5.7$ & 1.2 & 1.2 & $* * 2.9$ & Vatr2 \\
\hline CMM_2017 & Membrane protein, unknown function & $* 4.1$ & 1.3 & 1.2 & $* * 3.4$ & $* * 3.3$ & Vatr $1 / 2$ \\
\hline \multicolumn{8}{|c|}{ Metabolic proteins } \\
\hline $\begin{array}{l}\text { CMM_0120- } \\
\text { CMM_0123 }\end{array}$ & $\begin{array}{l}\text { Amino acid permease, } 2 \text { hypothetical } \\
\text { proteins, uahA urea amidolyase }\end{array}$ & $* 6.5$ & 0.6 & $* 0.1$ & $* * 2.8$ & $* * 9.0$ & Vatr1/2 \\
\hline CMM_0025 & RpmJ2 50S ribosomal protein & $* 2.4$ & $* 0.2$ & 0.7 & $* * 4.9$ & $* * 3.3$ & Vatr1/2 \\
\hline CMM_0206 & $\begin{array}{l}\text { 3-hydroxyacyl-Coenzyme A } \\
\text { dehydrogenase }\end{array}$ & $* 2.8$ & 0.6 & 0.3 & 1.9 & $* * 3.1$ & Vatr2 \\
\hline CMM_0211 & Monooxygenase & $* 21.8$ & $* 4.3$ & 2.5 & $* * 4.5$ & $* * 5.6$ & Vatr1/2 \\
\hline CMM_0303 & Short chain oxidoreductase & $* 4.5$ & 0.5 & 0.4 & $* * 3.5$ & 2.9 & Vatr1 \\
\hline CMM_0315 & Thioredoxin reductase & $* 243.2$ & $* 4.4$ & 2.3 & $* * 4.0$ & $* * 5.5$ & Vatr1/2 \\
\hline CMM_0334 & Organic acid synthase & *6.8 & 1.1 & 0.4 & $* * 2.4$ & $* * 4.3$ & Vatr $1 / 2$ \\
\hline CMM_1227 & Methyltransferase & $* 83.3$ & 3.2 & 3.4 & $* 6.4$ & $* * 5$ & Vatr1/2 \\
\hline CMM_1515 & Hhydrolase/acyltransferase & *6.9 & 0.8 & 0.6 & $* * 2.7$ & 1.7 & Vatr1 \\
\hline CMM_2025 & Recombinase A & $* 3.1$ & 1.4 & 1.7 & $* * 2.2$ & 1.9 & Vatr1 \\
\hline CMM_2361 & Acetyltransferase & $* 3.0$ & 0.9 & 1.0 & $* * 3.0$ & $* * 2.7$ & Vatr1/2 \\
\hline \multicolumn{8}{|c|}{ Reactive oxygen species } \\
\hline CMM_0426 & Manganese-containing catalase & $* 2.3$ & 1.1 & 0.7 & $* * 2.0$ & $* * 2.8$ & Vatr $1 / 2$ \\
\hline \multicolumn{8}{|c|}{ Hypothetical proteins } \\
\hline CMM_0178 & Unknown function & $* 7.7$ & 1.6 & 0.8 & $* * 3.3$ & $* * 7.6$ & Vatr $1 / 2$ \\
\hline CMM_0207 & Unknown function & $* 4.8$ & $* 0.3$ & $* 0.2$ & $* * 6.0$ & $* * 7.6$ & Vatr1/2 \\
\hline CMM_0210 & Unknown function & $* 4.5$ & 1.7 & 1.5 & $* * 2.7$ & $* * 2.6$ & Vatr1/2 \\
\hline CMM_0593 & Unknown function & $* 10.4$ & $* 7.9$ & 2.7 & 1.2 & $* * 2.7$ & Vatr2 \\
\hline CMM_1081 & Unknown function & *3.6 & 1.6 & 1.0 & $* * 2.1$ & 2.2 & Vatr1 \\
\hline CMM_1706 & Unknown function & $* 11.7$ & 2.4 & 0.9 & $* * 4.0$ & $* * 6.4$ & Vatr1/2 \\
\hline CMM_2043 & Unknown function & $* 5.5$ & 1.3 & 0.6 & $* * 2.0$ & $* * 2.3$ & Vatr1/2 \\
\hline CMM_2352 & Unknown function & $* 2.8$ & 2.0 & 0.9 & 1.7 & $* * 2.6$ & Vatr2 \\
\hline \multicolumn{8}{|l|}{ CMM_2681, } \\
\hline CMM_2682 & Unknown function & $* 2.2$ & 0.7 & 0.5 & 2.1 & $* * 3.9$ & Vatr2 \\
\hline CMM_2902 & Unknown function & $* 4.1$ & $* 5.3$ & 1.0 & 1.0 & $* * 2.4$ & Vatr2 \\
\hline pCM2_0001 & Unknown function & $* 7.5$ & 1.3 & 0.5 & $* * 2.0$ & $* * 3.8$ & Vatr $1 / 2$ \\
\hline pCM2_0059 & Unknown function & $* 2.2$ & 1.0 & 0.7 & $* * 2.3$ & $* * 4.8$ & Vatr1/2 \\
\hline
\end{tabular}

a Asterisks: * indicates that induction of gene expression in IMM was at least twofold higher than in LB, with a $P$ value $<0.05$, and $* *$ indicates that gene expression in IMM was at lest twofold higher in the wild-type Cmm382 than in the mutant, with a $P$ value $<0.05$.

${ }^{\mathrm{b}}$ Fold induction of gene expression between rich (LB) and infection-mimicking medium (IMM).

${ }^{\mathrm{c}}$ Ratio of gene expression in IMM between wild-type Cmm382 and the mutant. 
protein family is characterized by a conserved N-terminal HTH domain, followed by a C-terminal domain that is divergent among several subfamilies. Vatr2 belongs to the MocR subfamily of GntR proteins. Members of this subfamily contain a C-terminal domain of approximately 350 residues homologous to class I aminotransferase proteins, which are enzymes that catalyze the reversible transfer of an amino group from the amino acid substrate to an acceptor $\alpha$-keto acid, and require pyridoxal $5^{\prime}$-phosphate (PLP) as a cofactor for this reaction (Bramucci et al. 2011). Therefore, it is thought that MocR proteins also require PLP as a cofactor for their regulating activity (Rigali et al. 2002). The hypothesis that Vatr2 is a MocR protein that requires PLP as a co-factor is further supported by the fact that a multiple alignment of the Vatr2 amino acid sequence to other known MocR proteins indicates that all of the hypothetical PLP-binding residues are conserved in Vatr2 (Supplementary Fig. S3). The vatr 2 gene is genomically located directly downstream of the two thioredoxins genes trxB1 (CMM_2967) and trxA (CMM_2968). Thioredoxins are small redox proteins that reduce different molecules. Thus, in planta, TrxA and TrxB1 may reduce reactive oxygen species (ROS) that are produced as a part of the tomato defense response. Directly upstream of vatr2 is a D-alanine-D-alanine ligase gene (CMM 2970), whose product is involved in peptidoglycan biosynthesis and which may affect the survival of $C$. michiganensis subsp. michiganensis in planta by strengthening the bacterial cell wall, thus enhancing resilience against plant assault. Thus, the genomic localization of vatr2 suggests its possible involvement in counteracting plant defense.

\section{Identification of molecular pathways affected by Vatr1 and Vatr2.}

Transfer of $C$. michiganensis subsp. michiganensis from rich medium to the IMM minimal medium results in a protein expression profile similar to the one in planta (Savidor et al. 2012). Based on this observation, an RNA-seq experiment was

Table 2. List of genes negatively regulated by Vatr1 or Vatr2 in infection-mimicking conditions ${ }^{\mathrm{a}}$

\begin{tabular}{|c|c|c|c|c|c|}
\hline Category, locus & Annotations & $\begin{array}{c}C \mathrm{Cmm382} \\
(\mathrm{IMM} / \mathrm{LB})^{\mathrm{b}}\end{array}$ & $\begin{array}{l}\Delta v a t r 1 / C m m 382 \\
(\mathrm{IMM})^{\mathrm{c}}\end{array}$ & $\begin{array}{l}\Delta v a t r 2 / C m m 382 \\
\quad(\mathrm{IMM})^{\mathrm{c}}\end{array}$ & $\underset{\text { by }}{\text { Regulated }}$ \\
\hline \multicolumn{6}{|c|}{ Membrane or secreted enzymes } \\
\hline CMM_1126 & Putative 5'-nucleotidase & 0.6 & $* 6.9$ & 1.2 & Vatr1 \\
\hline CMM_0819 & WcoA cell surface protein & 1.1 & $* 3.6$ & 2.0 & Vatr1 \\
\hline CMM_2535 & SbtB serine protease & 1.8 & $* 2.7$ & 1.2 & Vatr1 \\
\hline CMM_1674 & XysB endo- $1,4-\beta$-xylanase & 1.0 & $* 2.5$ & 0.7 & Vatr1 \\
\hline CMM_0724 & WcnA membrane-bound acyltransferase & 0.7 & $* 2.0$ & 1.3 & Vatr1 \\
\hline CMM_1574,CMM_1575 & $\begin{array}{l}\text { Membrane bound esterase }+ \text { membrane bound } \\
\text { serine peptidase }\end{array}$ & 0.8 & 1.0 & $* 2.6$ & Vatr2 \\
\hline CMM_0638 & Membrane metalloendopeptidase, subfamily M23B & 1.0 & 1.2 & $* 2.2$ & Vatr2 \\
\hline \multicolumn{6}{|l|}{ Signal transduction } \\
\hline CMM_0678 & PknC serine/threonine-protein kinase & 0.6 & $* 2.4$ & 1.6 & Vatr1 \\
\hline CMM_0089 & CatR LacI-family transcriptional regulator & 0.4 & $* 2.4$ & 1.0 & Vatr1 \\
\hline CMM_0537 & Transcriptional regulator & 1.9 & $* 2.1$ & $* 4.4$ & Vatr $1 / 2$ \\
\hline CMM_1573 & Two-component system, response regulator & 0.9 & 1.0 & $* 4.0$ & Vatr2 \\
\hline CMM_1572 & Two-component system, sensor kinase & 1.0 & 1.0 & $* 3.7$ & Vatr2 \\
\hline CMM_0785 & Transcriptional regulator, GntR family & 0.8 & 1.3 & $* 2.9$ & Vatr2 \\
\hline CMM_1479 & Transcriptional regulator, TetR family & 1.1 & 1.6 & $* 2.7$ & Vatr2 \\
\hline CMM_2462 & Lsr2-like transcriptional regulation & 0.5 & 1.7 & $* 2.2$ & Vatr2 \\
\hline CMM_2390 & $\begin{array}{l}\text { Zinc finger domain containing protein } \\
\text { (DNA binding) }\end{array}$ & 2.0 & 1.0 & $* 2.2$ & Vatr2 \\
\hline \multicolumn{6}{|l|}{ Antibiotic metabolism } \\
\hline CMM_1968 & ClvM lantibiotic modifying enzyme & 1.4 & 0.8 & $* 5.0$ & Vatr2 \\
\hline CMM_1967 & ClvA lantibiotic precursor & 1.8 & 0.7 & $* 3.3$ & Vatr2 \\
\hline CMM_2676 & BlaC $\beta$-lactamase & 2.1 & 0.5 & $* 3.1$ & Vatr2 \\
\hline \multicolumn{6}{|l|}{ Others } \\
\hline CMM_2329 & Mannose-6-phosphate isomerase & 0.4 & $* 2.5$ & 2.3 & Vatr1 \\
\hline CMM_1315 & MFS-type efflux sugar transporter & 1.4 & $* 2.1$ & 1.0 & Vatr1 \\
\hline CMM_1422 & Acetyltransferase & 0.5 & $* 2.1$ & $* 3.0$ & Vatr1/2 \\
\hline CMM_2252 & Secreted protein with a ferritin-like domain & 1.5 & $* 2.0$ & 0.8 & Vatr1 \\
\hline CMM_1141 & Glutamine amidotransferase & 1.4 & 1.3 & *2.5 & Vatr2 \\
\hline CMM_1848 & HisD histidinol dehydrogenase & 1.1 & 1.3 & $* 2.1$ & Vatr2 \\
\hline CMM_0581 & General stress response protein & 0.9 & 1.0 & $* 2.6$ & Vatr2 \\
\hline CMM_2667 & Membrane protein with a ferritin-like domain & 2.4 & 0.5 & $* 8.5$ & Vatr2 \\
\hline CMM_1928 & Unknown function & 1.1 & $* 2.1$ & 1.6 & Vatr1 \\
\hline \multicolumn{6}{|l|}{ Hypothetical proteins } \\
\hline CMM_2757 & Unknown function & 0.4 & $* 2.2$ & 1.6 & Vatr1 \\
\hline pCM1_0006 & Unknown function & 2.2 & $* 2.1$ & 0.6 & Vatr1 \\
\hline CMM_2689 & Unknown function & 1.2 & $* 2.1$ & 1.4 & Vatr1 \\
\hline CMM_0556 & Unknown function & 0.8 & 1.1 & $* 11.9$ & Vatr2 \\
\hline CMM_2540 & Unknown function & 1.5 & 0.6 & $* 4.7$ & Vatr2 \\
\hline CMM_0784 & Unknown function & 1.7 & 0.6 & $* 4.0$ & Vatr2 \\
\hline CMM_1610 & Unknown function & 0.7 & 2.4 & $* 3.5$ & Vatr2 \\
\hline CMM_1230 & Unknown function & 2.3 & 0.7 & $* 3.4$ & Vatr2 \\
\hline CMM_2857 & Unknown function & 1.4 & 0.9 & $* 3.0$ & Vatr2 \\
\hline CMM_0773 & Unknown function & 0.8 & 1.9 & $* 2.6$ & Vatr2 \\
\hline CMM_2712 & Unknown function & 1.0 & 2.0 & $* 2.5$ & Vatr2 \\
\hline CMM_2444 & Unknown function & 0.7 & 0.8 & $* 2.4$ & Vatr2 \\
\hline
\end{tabular}

a Asterisk (*) indicates that gene expression in IMM was at lest twofold higher in the mutant than in the wild-type $C m m 382$ with a $P$ value $<0.05$.

${ }^{\mathrm{b}}$ Fold induction of gene expression between rich (LB) and infection-mimicking medium (IMM).

c Ratio of gene expression in IMM between the mutant and wild-type Cmm382. 
designed on a series of in vitro $C$. michiganensis subsp. michiganensis samples to identify the molecular pathways affected by Vatr1 and Vatr2. Before the samples were prepared, we attempted to identify the time point in which the expression of vatr 1 , vatr 2 , and of the pat- 1 virulence gene is the most significant. To this aim, Cmm382 was grown in LB medium to midexponential phase, and transferred to IMM. Total RNA was extracted from aliquots of cells that were harvested at time 0, 30, 180, and 360 min post-transfer. Expression of the vatrl, vatr2, and pat-1 genes in these RNA samples was monitored by quantitative (q)PCR. The expression of pat-1 and vatrl was highest at $180 \mathrm{~min}$ post-transfer to IMM, whereas expression of vatr2 did not significantly change after transfer to IMM along the timeline, and even slightly decreased (Fig. 5). Based on these results, for the RNA-seq sample preparation, Cmm382, $\Delta$ vatrl, and $\Delta$ vatr 2 bacteria were grown in LB, transferred to IMM, and harvested after $180 \mathrm{~min}$. Aliquots of cultures in LB medium before transfer were also harvested. Three biological replicates of wild-type and mutant cultures growing in parallel were prepared. Total RNA was extracted from all samples and was used for RNA-seq analysis. Expression profiles for each strain were quantitatively compared between LB and IMM to identify differentially expressed genes between treatments. Quantitative comparisons were also made between the wild type and mutants in either of the media to identify differentially expressed genes between strains.

In agreement with our previous proteomic study (Savidor et al. 2012), the majority of genes encoding secreted hydrolytic enzymes were upregulated in $C m m 382$ after transfer to IMM (Supplementary Fig. S4), indicating a consistent pattern of virulence regulation at the transcript and protein level.

As transcriptional regulators, Vatr1 and Vatr2 are expected to positively or negatively regulate expression of other genes, either directly or indirectly. If a transcriptional regulator is virulence specific, the genes that are positively regulated by it are expected to be upregulated in IMM in the wild-type Cmm382. In the mutant of that transcriptional regulator, expression of these target genes is not expected to change (or the magnitude of change is expected to be significantly lower, if other factors cumulatively contribute to the regulation). Therefore, to identify genes that are positively regulated by Vatr1 or Vatr2, we looked for genes that are upregulated in IMM in the wild-type $C m m 382$ but not (or to a lesser extent) in the mutants, and for genes that, in IMM, are expressed higher in Cmm382 than in the mutants. In all, 34 such genes were identified for Vatr1 and 36 were identified for Vatr2 (Table 1). Interestingly, 25 genes (approximately 70\%) were commonly regulated by Vatr1 and Vatr2, and most of the other genes displayed a similar regulation pattern, although they did not pass all dif-

Fig. 6. Timeline transcriptional expression in planta of the pat-1, celA, vatr1, and vatr 2 genes in wild-type and mutant Clavibacter michiganensis subsp. michiganensis strains. A, Tomato plants were inoculated with wildtype Cmm382. Total RNA was extracted from the infected plants at different hours postinoculation (hpi) and was analyzed by quantitative reverse-transcriptase polymerase chain reaction (qRT-PCR) with vatrl and vatr2 gene-specific primers, using gyrA and bipA as normalizers. $\mathbf{B}$ to $\mathbf{D}$, Tomato plants were inoculated with wild-type Cmm382, $\Delta v a t r 1$, or $\Delta$ vatr2. Total RNA was extracted from the infected plants at different hours postinoculation and was analyzed by qRT-PCR with $\mathbf{B}$, vatrl or vatr 2 ; $\mathbf{C}$, pat-1; and $\mathbf{D}$, celA gene-specific primers, using gyrA and bipA as normalizers. Results represent the mean of three (in A, C, and D) or two (in B) biological repeats with standard deviation. Asterisks (*) represent statistically significant differences $(P$ value $<0.05)$ in the expression of the examined genes at the indicated time points, compared with $0 \mathrm{~h}$ postinoculation in A and compared with $\mathrm{Cmm} 382$ in B, C, and D, according to a $t$ test with $P$ value $<0.05$. ferential expression criteria (see Materials and Methods). This suggests that the two transcriptional regulators share a common pathway, and that a large group of positively regulated genes are downstream of both. Among the positively regulated

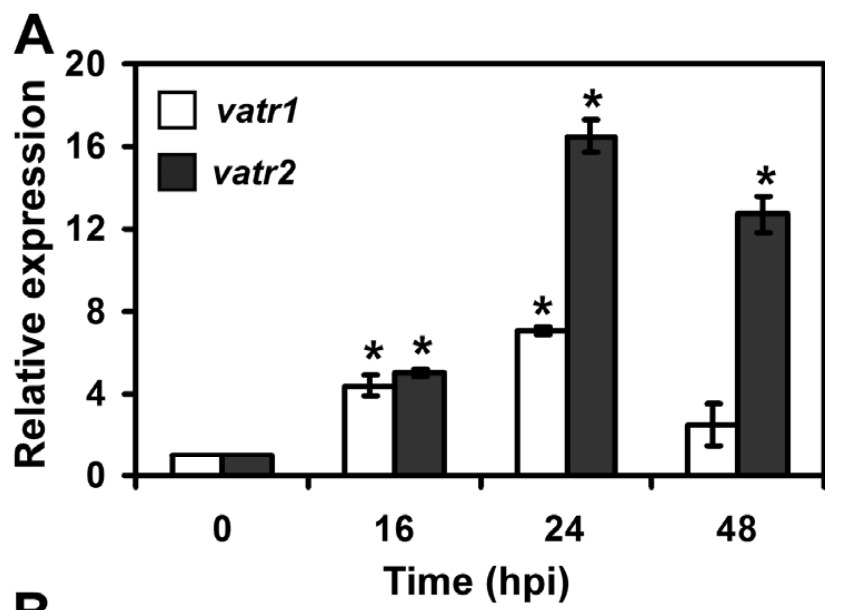

B
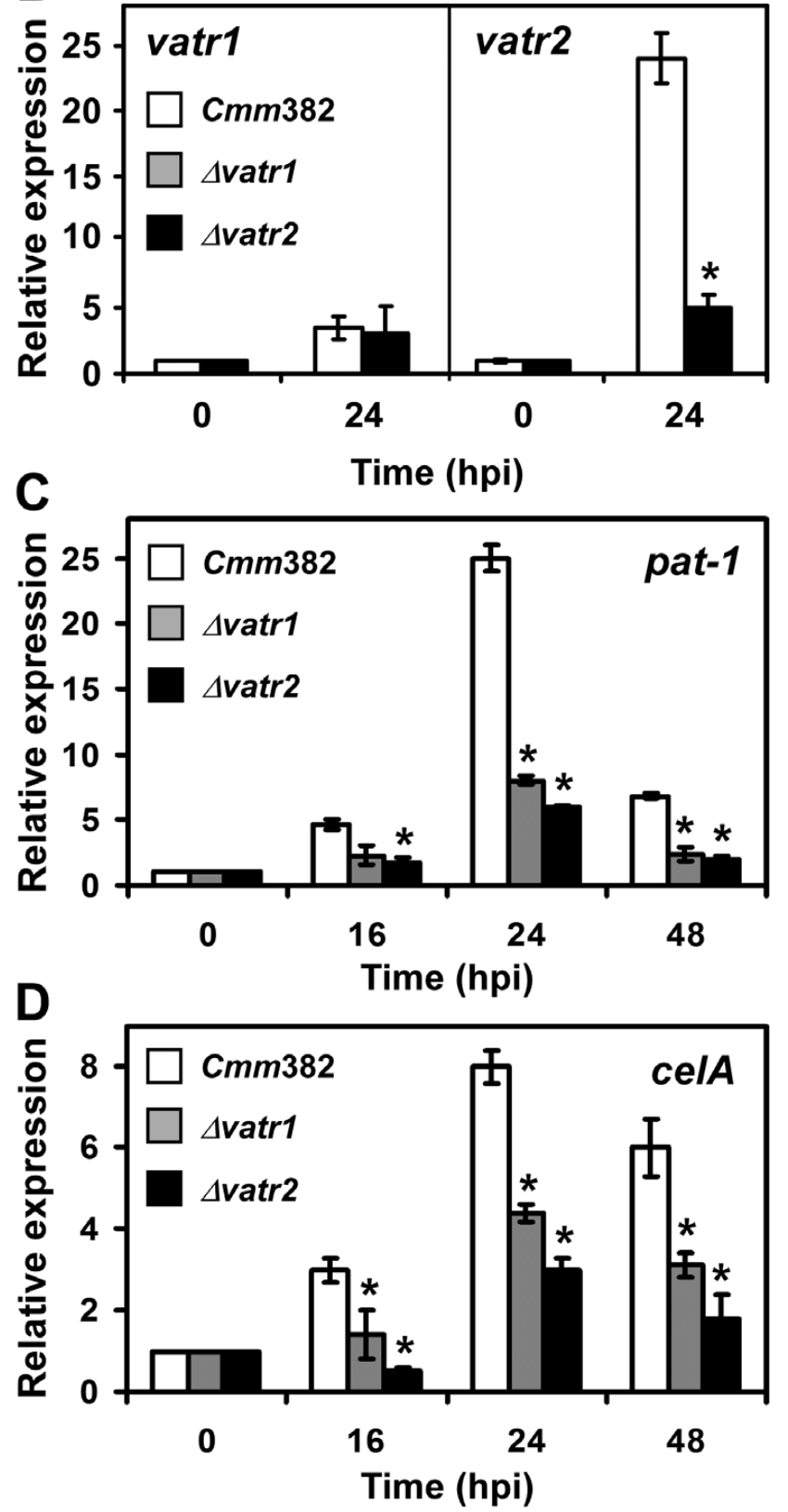
genes are several virulence factors, including pat-1, one of the most important known virulence factors of $C$. michiganensis subsp. michiganensis, as well as the pat-1 homolog phpA (Table 1). The reduced expression of pat-1 in the mutants was also confirmed by qPCR (Supplementary Fig. S5). The operon including the four genes CMM_0328 to CMM_0331, which encodes genes responsible for the biosynthesis of a siderophore homologous to the biosynthetic genes of the Yersinia virulence factor Yersiniabactin (Ybt), is also commonly regulated by Vatr1 and Vatr2. Ybt is a siderophore found in pathogenic Yersinia spp. that is crucial for iron uptake in the host and plays an important role in pathogenicity (Perry and Fetherston 2011). A group of regulatory genes also positively regulated by Vatr1 and Vatr2 encodes four transcriptional regulators from three different families (GntR, TetR, and MarR), as well as two phosphatases. Other genes positively regulated by Vatr1 and Vatr2 encode the chaperones ClpY and GroEL, the heat-shock protein CMM_2092, several membrane proteins, proteins involved in metabolism, a catalase involved in ROS scavenging, and several hypothetical proteins with unknown function.

In agreement with the preliminary qPCR experiments (Fig. 5), the RNA-seq results indicated that, in the wild type, vatrl was upregulated after transfer to IMM (although upregulation was 2.3 -fold with a $P$ value of 0.11 ), whereas vatr 2 expression did not change. In addition, RNA-seq and qPCR analysis of in vitro samples revealed no significant effect on the expression of vatrl transcript in the $\Delta$ vatr 2 mutant, and vice-versa.

To identify genes that are negatively regulated by Vatr1 or Vatr2 in infection-mimicking conditions, we looked for genes that are not upregulated in the wild type after transfer to IMM, and that are expressed higher in the mutants than in the wild type in IMM. In total, 16 such genes were identified for Vatr1, almost all of which (14 genes) were expressed only moderately higher in the mutants than in the wild type (less than threefold) (Table 2). On the other hand, 26 genes were identified for Vatr2, half of which were expressed markedly higher in the mutants than in the wild type (threefold or more) (Table 2). Among all of the genes that were negatively regulated by Vatr1 or Vatr2, only two genes, an acetyltransferase and a transcriptional regulator, showed similar regulation patterns by both. Together, these results suggest that, among the two proteins, Vatr2 is the major negative transcriptional regulator.

Interestingly, of the 40 genes negatively regulated by Vatr1 and Vatr2, most genes (23 genes) encode either membrane or secreted proteins. These include hydrolytic enzymes, an acetyltransferase, an esterase, a sugar transporter, and several proteins of unknown function. Another prominent group of negatively regulated genes encodes proteins involved in signal perception and transduction, most of which are uniquely regulated by Vatr2 (Table 2). These include transcriptional regulators from different families, a protein kinase, and a complete two-component system (sensor kinase and its response regulator). A group of three enzymes involved in antibiotics metabolism was also uniquely negatively regulated by Vatr2.

Expression of vatrl and vatr2 in wild-type C. michiganensis subsp. michiganensis and in the mutants was also monitored in planta by qPCR. In agreement with the in vitro analysis, in planta, both genes were transcriptionally upregulated during infection in wild-type $C$. michiganensis subsp. michiganensis (Fig. 6A). However, although deletion of vatr2 had no effect on the expression of vatrl, in the $\Delta v a t r l$ mutant, expression of vatr2 was significantly reduced compared with wild-type $C$. michiganensis subsp. michiganensis in infected plants (Fig. 6B). Similar qPCR experiments carried out on plants infected

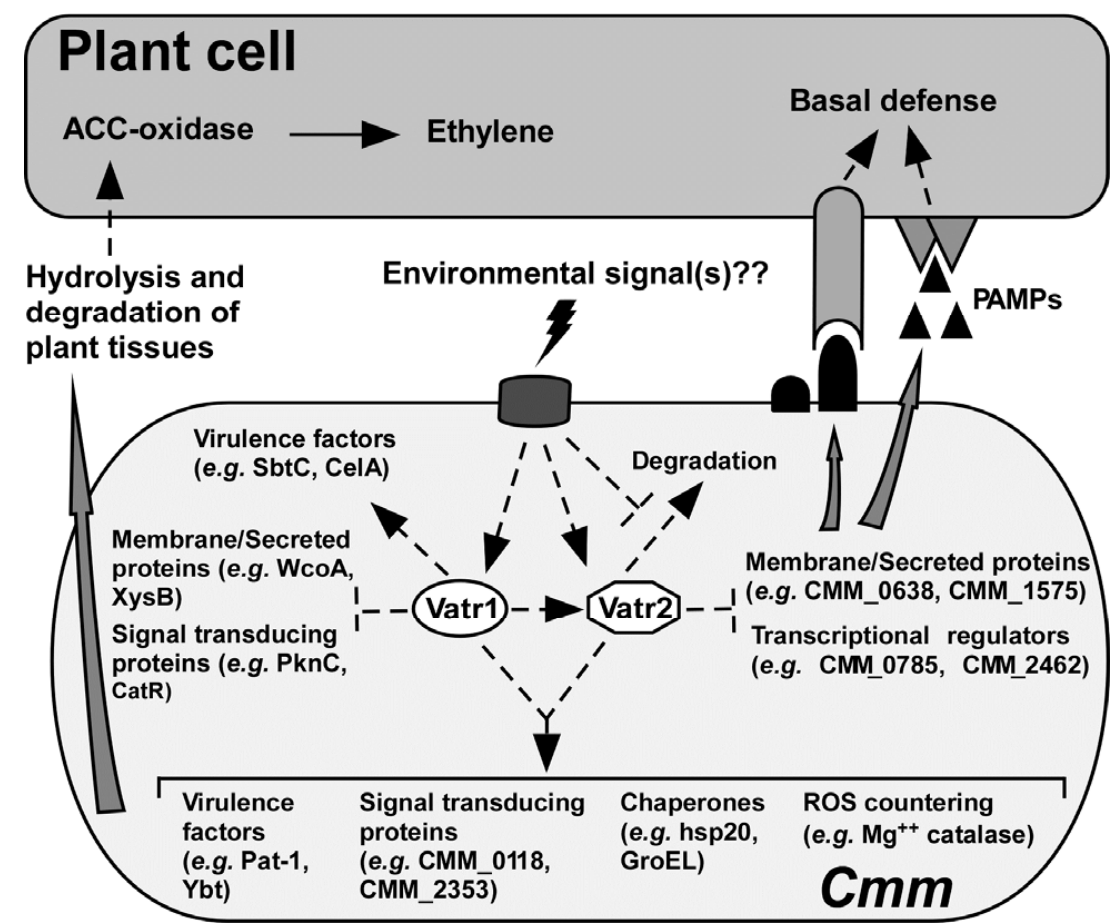

Fig. 7. Proposed model for Clavibacter michiganensis subsp. michiganensis pathogenicity. Upon invasion, C. michiganensis subsp. michiganensis senses the plant environment and transduces a signal for activation of a virulence pathway. Induction of the virulence pathway leads to transcriptional activation of vatr1 and vatr2 as well as to increased stability of the Vatr2 protein. Vatr1 and Vatr2 regulate downstream genes in the pathway, either directly by binding their regulatory DNA region or indirectly by affecting other transcriptional regulators that regulate them. Vatr1 and Vatr2 are found on separate branches of the virulence pathway that converge at Vatr2 and, possibly, elsewhere in the pathway. Upstream of their convergence in the pathway, expression of genes coding for secreted and membrane proteins, which may be recognized by the plant as pathogen-associated molecular patterns (PAMPs) and induce a defense response, is downregulated. Nonetheless, downstream of the convergence in the pathway, genes involved in virulence, including several virulence factors, are upregulated. As a result, $C$. michiganensis subsp. michiganensis overcomes the plant defense response, hydrolyzes and degrades the plant tissue, and upregulates tomato ACC-oxidase and ethylene biosynthesis, thus resulting in stem canker and leaf wilting. 
with the wild type or mutants showed that inactivation of either vatrl or vatr2 led to a reduction in expression of the pat-1 and celA virulence factors (Fig. 6C and D).

Because multiple genes were regulated by Vatr1 and Vatr2, the promoter regions of these regulated genes were searched for enriched sequence motifs that may indicate a common binding site for their transcriptional regulators. This analysis revealed three and seven such potential binding motifs in genes regulated by Vatr1 and Vatr2, respectively (Supplementary Fig. $\mathrm{S} 6 \mathrm{~A}$ and $\mathrm{B})$.

\section{DISCUSSION}

In a previous proteomic study, we identified several candidate proteins for playing a role in $C$. michiganensis subsp. michiganensis pathogenicity. Here, we found that inactivation of the genes of two of them, Vatr1 and Vatr2, resulted in mutant strains that were less virulent than the wild type. The hypothesis that Vatr1 and Vatr2 are virulence-specific transcriptional regulators was supported by the following findings: i) $\Delta$ vatrl and $\Delta$ vatr 2 mutants were less virulent than the wild type, ii) Vatr1 and Vatr2 were not involved in general housekeeping processes (i.e., the reduced virulence of the mutants is not a consequence of their inability to grow in planta), and iii) Vatr1 and Vatr2 regulated expression of genes involved in pathogenicity.

To test virulence of the $\Delta v a t r 1$ and $\Delta v a t r 2$ mutants, tomato plants infected with the mutants or with wild-type Cmm382 were monitored for development of disease symptoms. Plants infected with either mutant displayed reduced disease symptoms compared with the wild type in the timing and extent of leaf wilting and, for $\Delta v a t r 2$, also in the size of stem canker. Among the two, the $\Delta v a t r 2$ mutant exhibited a more dramatic phenotype, resulting in no wilting in most of the infected plants. At the molecular level, many genes were positively coregulated by Vatr1 and Vatr2, as was evident from their reduced expression levels in the mutants when compared with the wild type in infection-mimicking conditions. These positively regulated genes encode proteins that include virulence factors, such as Pat-1, PhpA, and a siderophore-biosynthesis operon, as well as several transcriptional regulators, chaperones, membrane proteins, and hypothetical proteins. Only a small number of genes were positively regulated by one of the two transcriptional regulators ( 9 genes by Vatr1 and 11 genes by Vatr2) but not by both.

Because C. michiganensis subsp. michiganensis-induced ethylene production via specific upregulation of the tomato ACO enzyme was shown to be correlated to disease symptom development (Savidor et al. 2012), both ethylene and ACO levels were monitored in plants infected with the mutants or with wildtype Cmm382. In correlation with disease symptom severity, plants infected with the $\Delta v a t r l$ mutant strain displayed a moderate reduction in ACO and ethylene levels, whereas plants infected with the $\Delta$ vatr 2 mutant showed a dramatic reduction in both. Taken together, it appears that Vatr1 and Vatr2 are located somewhere upstream in the same complex molecular pathway that leads to virulence, via signal transduction, upregulation of virulence factors and tomato $\mathrm{ACO}$, and in planta ethylene production which leads to symptom development.

Originally, Vatr1 and Vatr2 were identified as candidates for involvement in virulence based on their accumulation in vitro in IMM and in planta during infection, as detected by proteomic analysis (Savidor et al. 2012). Here, it was shown that, at the transcript level, both genes were upregulated in planta during infection in wild-type Cmm382. Surprisingly, in IMM, only vatr 1 was upregulated, while the vatr 2 transcript level did not change. Nevertheless, the $\Delta v a t r 2$ mutant still showed simi- lar characteristics in vitro to those in planta, including reduced expression of virulence factors and moderately reduced growth rate in IMM. This suggests that the Vatr2 protein still accumulates and performs its downstream regulatory action in wild-type $C$. michiganensis subsp. michiganensis in infectionmimicking conditions, despite the lack of vatr2 transcript accumulation. Together, proteomic and RNA-seq analysis suggest that, in addition to the transcriptional regulation shown in planta, Vatr2 is regulated post-translationally, because its stability is increased in IMM and in planta during infection.

Both mutants grew as well as the wild type in LB medium, suggesting that Vatr1 and Vatr2 are not involved in essential housekeeping processes. Deletion of vatr2 somewhat impaired the ability of $C$. michiganensis subsp. michiganensis to grow in planta and in IMM. Thus, Vatr2 may also influence some metabolic pathway during infection. Because $\Delta v a t r 2$ titer in planta is lower than that of wild-type $C$. michiganensis subsp. michiganensis, it is possible that some of the reduction in symptom development, ACO expression, and ethylene emission is due to the reduced bacterial number and not solely to reduced virulence. Nevertheless, the $\Delta v a t r 2$ mutant still grew to a very high titer in planta, and the moderate reduction in growth by itself is not likely to account for the dramatic reduction in virulence of the mutant. This suggests a significant role for Vatr2, specifically, in C. michiganensis subsp. michiganensis pathogenicity.

Although direct DNA binding by Vatr1 and Vatr2 has not been experimentally demonstrated, indirect evidence suggests that this is, indeed, the case. Bioinformatic analysis indicated that both proteins contain a helix-loop-helix DNA-binding domain and belong to families of DNA-binding proteins. Specifically, Vatr1 is classified into the TetR family of transcriptional repressors and Vatr2 is classified into the MocR subfamily of GntR transcriptional regulators with high confidence. Proteins of the TetR family have not only been shown to be DNA-binding transcriptional repressors but also were also implicated in regulating virulence. For example, the TetR family member HapR from Vibrio cholerae has been shown to be a transcriptional regulator with a central role in control of virulence, in a cell-density-dependent way (Kovacikova and Skorupski 2002), and SmcR from $V$. vulnificus regulates the hemolysin and metalloprotease virulence factors (Choi et al. 2002; Shao and Hor 2001). Results from the RNA-seq experiments also clearly show that expression of several groups of genes, including known and putative virulence factors, is regulated by Vatr1 or Vatr2, either directly or indirectly. Both proteins regulated other transcriptional regulators, suggesting that they occupy a high position in the hierarchy of virulence regulation. Bioinformatic analysis identified several potential common DNAbinding motifs in promoters of genes regulated by Vatr1 or Vatr2, and these should be examined experimentally for binding their respective transcriptional regulator.

As is evident from analysis of the RNA-seq results, the target genes regulated by Vatr1 and Vatr2 displayed an interesting pattern of regulation. Twenty-one genes, including putative virulence factors, transcriptional regulators, and chaperones, were positively coregulated by both transcriptional regulators in infection-mimicking conditions, while only a few other genes were positively regulated uniquely by one of them (Table 1). This suggests that Vatr1 and Vatr2 are a part of the same molecular pathway responsible for virulence, upstream of the positively regulated genes. Interestingly, in planta, Vatr1 was shown to contribute to vatr2 upregulation but not vice versa, which suggests a convergence point of the Vatr1 and Vatr2 branches in the pathogenicity pathway. It should be noted that Vatr2 expression is not completely abolished in the $\Delta v a t r 1 \mathrm{mu}-$ tant, and that the more dramatic reduction in virulence in the 
$\Delta v a t r 2$ mutant is probably the result of a complete abolishment of this protein. This may explain the fact that deletion of vatrl has a lesser effect on virulence than deletion of vatr2, even though Vatr1 is a positive regulator of Vatr2.

Unlike for the positively regulated genes, almost no overlap was observed between the sets of genes negatively regulated in infection-mimicking conditions by Vatr1 and Vatr2 (Table 2). This may suggest that Vatr1 and Vatr2 are found on separate branches of a signal transduction pathway that converge at a certain point (at or upstream to Vatr2) and positively regulate a subset of genes.

Regardless of which transcriptional regulator downregulated the negatively regulated genes, almost all were classified as membrane or secreted proteins or as signal transducers. Once again, the fact that other genes involved in signal transduction and transcriptional regulation are regulated by them indicates that Vatr1 and Vatr2 are found high in the hierarchy of a complex signal transduction pathway leading to virulence. Thus, the fact that many genes are positively regulated by vatrl, even though it is a predicted repressor, may be explained by its effect on other transcriptional regulators and signal transducers, which may have a negative effect on virulence. Although the exact function of many of them is unknown, the fact that secreted and membrane proteins were repressed during infection is particularly interesting, because they are found in the interface between the bacterium and the host plant. It is tempting to speculate that these proteins can be perceived by the plant and induce a defense response, and that their repression leads to a less effective plant defense response, as was suggested to be the case for $C$. michiganensis subsp. michiganensis-infected tomato plants (Savidor et al. 2012).

Based on all of the results, we propose a model (Fig. 7) in which, upon plant invasion, C. michiganensis subsp. michiganensis senses the new environment and transduces a signal for activation of a virulence pathway. Vatr1 and Vatr2 are on separate branches of this pathway that converge at Vatr2 and possibly elsewhere. Activation of the virulence pathway leads to transcriptional upregulation of vatrl and vatr 2 and to increased stability of the Vatr2 protein. Vatr1 and Vatr2 regulate downstream genes either directly by binding their regulatory DNA region or indirectly by regulating other transcriptional regulators that affect them. Vatr1 and Vatr2 downregulate expression of genes coding for secreted and membrane proteins, which may be recognized by the plant and induce a defense response. On the other hand, they upregulate a variety of genes that contribute to virulence, including several virulence factors (e.g., secreted proteases and the Ybt-synthesis-operon homolog), chaperones (e.g., clpY, groEL, and hsp20), metabolic genes, and other transcriptional regulators. The overall outcome is a pathogenic bacterium that can overcome the plant defense response, hydrolyze and degrade the plant tissue, and induce tomato ACO and ethylene biosynthesis, resulting in stem canker and leaf wilting. Further work will elucidate the direct targets of Vatr1 and Vatr2 regulation and map their position in the virulence pathway.

\section{MATERIALS AND METHODS}

\section{Bacterial strains, plasmids, and media.}

C. michiganensis subsp. michiganensis strains were grown in either LB medium (tryptone at $10 \mathrm{~g} /$ liter, yeast extract at 5 $\mathrm{g} /$ liter, and $\mathrm{NaCl}$ at $10 \mathrm{~g} /$ liter) or in tomato-sap-supplemented (1\%) minimal M9 medium (IMM) (Chalupowicz et al. 2010) at $28^{\circ} \mathrm{C}$. For genetic manipulation, after electroporation, competent $C$. michiganensis subsp. michiganensis cells were grown at $28^{\circ} \mathrm{C}$ on SB medium (Kirchner et al. 2001) supplemented with chloramphenicol $(10 \mu \mathrm{g} / \mathrm{ml})$ or neomycin $(75 \mu \mathrm{g} / \mathrm{ml})$. Escherichia coli DH12S was used for cloning and grown at $37^{\circ} \mathrm{C}$ in LB medium supplemented with ampicillin $(100 \mu \mathrm{g} / \mathrm{ml})$ or kanamycin $(100 \mu \mathrm{g} / \mathrm{ml})$.

Plasmids cmis3p0008c08 and cmis3p0003h08 (pSMART derivatives; Lucigen, Middleton, WI, U.S.A.) carrying the vatrl and vatr2 genes, respectively, along with pBlueScriptII KS(+) (pBS) (Agilent Technologies, Santa Clara, CA, U.S.A.), were used for construction of mutagenesis vectors. All three plasmids are unable to replicate in $C$. michiganensis subsp. michiganensis. For construction of the vatrl mutagenesis vector, plasmid cmis3p0008c08 was digested with EcoRV and $M s c$ I and resolved on an agarose gel. A 2.4-kb fragment containing the entire vatrl sequence was isolated and ligated to a $P v u I I-d i g e s t e d ~ p B S$. After transformation into E. coli and plasmid isolation, the pBS:vatrl plasmid was digested with SmaI, thereby deleting part of the gene, and ligated to a blunt chloramphenicol resistance ( $\mathrm{cm} x$ ) cassette produced by BsaAI digestion of the pEC70 plasmid (Tauch et al. 1998).

For construction of the vatr2 mutagenesis vector, plasmid cmis3p0003h08 was digested with $B g l \mathrm{II}$ and ligated to a 1.5$\mathrm{kb}$ Bam HI $\mathrm{cm} x$ cassette from plasmid pOKU9 $\mathrm{cmB} \alpha$, a derivative of pKGT452C $\beta$ (Gartemann and Eichenlaub 2001). Mutagenesis vectors were electroporated into competent Cmm382 cells as described (Kirchner et al. 2001). Transformants were screened for integration of the $c m x$ cassette into the correct site by PCR with diagnostic primers for vatrl and vatr2, as well as for the presence of the pCM2 plasmid, which is sometimes lost during the electroporation, with pat- 1 diagnostic primers.

The complementation vector for vatrl was constructed by ligation of the blunt 2.4-kb fragment described above into an EcoRV-digested pHN216 vector (Laine et al. 1996), which is able to replicate in $C$. michiganensis subsp. michiganensis and E. coli. The vatr 2 complementation vector was constructed by ligating the 2.7-kb fragment resulting from digestion of plasmid cmis3p0003h08 with BsaAI into an EcoRV-digested pHN216 vector.

For complementation assays, curing of pCM2 from $\Delta$ vatrl and $\Delta$ vatr 2 strains was done by picking and restreaking a single bacterial colony on LB agar plates three consecutive times, then screening for colonies that lost $\mathrm{pCM} 2$ by PCR of the pCM2-encoded pat-1 gene with the pat-1 diagnostic primers. Competent pCM2-cured cells of the mutants were generated and electroporated with complementation vectors. Presence of the complementation plasmid was confirmed in neomycinresistant colonies by PCR of vatrl or vatr2 with their respective diagnostic primers.

\section{Plant growth and inoculation.}

Tomato (S. lycopersicum) plants of the line 'Rio Grande' (Pedley and Martin 2003) were grown using standard greenhouse practices $\left(25^{\circ} \mathrm{C}\right.$, watering as needed, regime of $16 \mathrm{~h}$ of light and $8 \mathrm{~h}$ of darkness). Five-week-old plants were inoculated by pricking the stem between the cotyledons with a needle after picking a colony from 4-day-old $C$. michiganensis subsp. michiganensis plates or after dipping in water (control).

\section{Virulence assays.}

For the wilting index assay, 24 inoculated plants for each biological replicate were monitored for wilting of the first leaf. For the canker development assay, 15 days after inoculation, the stem of 15 infected plants for each treatment was cut and photographed. Canker area size was measured using the software ImageJ. Three biological replicates were prepared and analyzed for each assay.

\section{Southern blot.}

A $1.9-\mathrm{kb}$ probe for vatrl and a $1.2-\mathrm{kb}$ probe for vatr2 were created by PCR amplification from Cmm382 genomic DNA 
with sequence-specific primers. The respective amplicons were labeled with digoxygenin-11-dUTP using the DIG-high prime DNA labeling and detection starter kit I (Roche Applied Science, Mannheim, Germany) according to the manufacturer's instructions. For total DNA isolation, wild-type Cmm382, $\Delta v a t r 1$, and $\Delta v a t r 2$ were grown in $20 \mathrm{ml}$ of LB medium overnight and $4.5 \mathrm{ml}$ of culture was harvested by centrifugation. Cell pellets were suspended in $1 \mathrm{ml}$ of lysozyme buffer (20 $\mathrm{mM}$ Tris-HCl, 2 mM EDTA, and 1\% Triton X-100) and treated with lysozyme at $1 \mathrm{mg} / \mathrm{ml}$ at room temperature for $1 \mathrm{~h}$. Lysis buffer $(500 \mu \mathrm{l})(1 \%$ sodium dodecyl sulfate [SDS], $50 \mathrm{mM}$ Tris [pH 7.5], and $250 \mathrm{mM}$ EDTA) was then added and the mix was incubated at $37^{\circ} \mathrm{C}$ for $30 \mathrm{~min}$. DNA was recovered from lysed samples using phenol/chloroform extraction and ethanol precipitation. DNA pellets were suspended in TrisEDTA buffer, and $1 \mu \mathrm{g}$ of each DNA was digested with $N c o$ I and separated on a $1 \%$ agarose gel. The DNA was subsequently transferred to a Hybond-N nylon membrane (Amersham, Piscataway, NJ, U.S.A.) and crosslinked by UV. Hybridization against the labeled vatrl and vatr 2 probes was carried out at $68^{\circ} \mathrm{C}$ overnight in a buffer containing $5 \times \mathrm{SSC}(1 \times \mathrm{SSC}$ is 0.15 $\mathrm{M} \mathrm{NaCl}$ and $0.015 \mathrm{M}$ sodium citrate), $0.02 \% \mathrm{SDS}, 0.1 \% \mathrm{Na}-$ laurylsarcosyl, and 2\% blocking reagent (Roche Applied Science). The nylon membrane was washed twice in $2 \times$ SSC and $0.1 \%$ SDS at room temperature for $5 \mathrm{~min}$ each time, and once in $0.1 \times \mathrm{SSC}$ and $0.1 \% \mathrm{SDS}$ at $68^{\circ} \mathrm{C}$ for $20 \mathrm{~min}$. Detection was carried out as recommended by the manufacturer (Roche Applied Science).

\section{In vitro growth assay.}

Cmm382, $\Delta$ vatrl, and $\Delta v a t r 2$ cultures were grown in 100-ml flasks in $20 \mathrm{ml}$ of $\mathrm{LB}$ medium at $28^{\circ} \mathrm{C}$ overnight and harvested by centrifugation. For growth assay in rich medium, the cell pellet was resuspended in fresh LB medium and an aliquot was used to inoculate a fresh $20 \mathrm{ml}$ of LB medium, such that optical density at $600 \mathrm{~nm}\left(\mathrm{OD}_{600}\right)$ was 0.02 . For growth assays in infection-mimicking conditions, the overnight culture cell pellet was resuspended in IMM medium, and an aliquot was used to inoculate a fresh $20-\mathrm{ml}$ IMM such that $\mathrm{OD}_{600}$ was 0.2 (a lower inoculum does not grow well in IMM). Cultures were grown at $28^{\circ} \mathrm{C}$ and $\mathrm{OD}_{600}$ was monitored throughout the experiments. Three separate cultures were prepared for each strain in each condition. Three biological replicates of the entire experiment were performed to verify reproducible results.

\section{In planta growth assay.}

Five-week-old tomato plants were inoculated with $\mathrm{Cmm} 382$, $\Delta v a t r 1$, or $\Delta v a t r 2$. For each strain, a $0.5-\mathrm{cm}$ stem section from 10 inoculated plants was cut at $1 \mathrm{~cm}$ above the inoculation site at $4,7,13$, and 15 days postinoculation. The stem section was homogenized in $400 \mu \mathrm{l}$ of sterile water and serially diluted, and $5 \mu \mathrm{l}$ was plated on LB agar plates. Colonies were allowed to grow for 4 to 5 days and then counted, followed by calculation of original CFU concentration. At least two biological replicates were performed for each time point for each strain.

\section{Ethylene emission.}

Ethylene measurements were conducted as previously described by Balaji and associates (2008). Briefly, 5-week-old Rio Grande tomato plants were infected with Cmm382, $\Delta v a t r 1, \Delta v a t r 2$, or sterile water (mock inoculation). For each treatment, $1-\mathrm{cm}$ long stem sections were excised from 15 independent plants at $1 \mathrm{~cm}$ above the inoculation site, weighed, and distributed among three $25-\mathrm{ml}$ flasks. Following a 3 -h period during which the stem pieces were left uncovered to allow ethylene produced in response to the wounding to escape, the flasks were sealed for $4 \mathrm{~h}$, and 1-ml gas samples were removed with a syringe and measured for ethylene content with a Varian 3350 gas chromatograph (Varian Inc., Palo Alto, CA, U.S.A.).

\section{Western blotting analysis.}

Five-week-old Rio Grande tomato plants were inoculated with Cmm382, svatr1, $\Delta$ vatr2, or sterile water (mock inoculation). Eight days postinoculation, stem sections were harvested and total protein was extracted and analyzed by Western blot with anti-ACO antibodies as previously described (Savidor et al. 2012).

\section{Bioinformatics.}

Homology searches were done using BlastP. Domain searches were done using Pfam. Protein sequence alignment for Vatr2 was done using ClustalX against other MocR proteins, as described (Bramucci et al. 2011). Promoter analysis for potential Vatr1- and Vatr2-binding motifs was done using the Amadeus software (Linhart et al. 2008) using the promoter sequences (300 bp upstream to the start codon) of Vatr1- or Vatr2-regulated genes as inputs, separately. These sequences were searched for 10-bp-long motifs that were enriched in the specified dataset compared with a background list containing the promoter sequence of all C. michiganensis subsp. michiganensis genes.

\section{RNA isolation.}

Cultures of Cmm382, $\Delta v a t r 1$, and $\Delta$ vatr2 were grown in parallel overnight in LB medium and were used to inoculate $20 \mathrm{ml}$ of fresh $\mathrm{LB}$ medium to $\mathrm{OD}_{600}=0.3$. Cultures were allowed to grow to $\mathrm{OD}_{600}=0.6$. An aliquot of each culture was harvested by centrifugation and immediately frozen in liquid nitrogen (this sample was considered time 0) while the rest of the samples was harvested by centrifugation and used to inoculate fresh IMM cultures. For timeline experiments, aliquots from a Cmm382 IMM culture were harvested by centrifugation at different time points $(0,30,180$, and $360 \mathrm{~min})$ and immediately frozen in liquid nitrogen. For RNA-seq samples, wildtype and mutant IMM cultures were harvested 180 min after culture inoculation. Frozen pellets were stored at $-80^{\circ} \mathrm{C}$ until RNA extraction. RNA was extracted from the cell pellets according to the triton/chloroform protocol, as described (Rivas et al. 2001). Nucleic acid pellets were resuspended and treated with DNAse I (Promega Corp., Madison, WI, U.S.A.) in digestion buffer (10 mM Tris-HCl [pH 7.6], $2.5 \mathrm{mM} \mathrm{MgCl}_{2}$, and 0.5 $\mathrm{mM} \mathrm{CaCl}_{2}$ ) for $20 \mathrm{~min}$ at room temperature. RNA was then ethanol precipitated and resuspended in nuclease-free water for further applications. Three biological replicates were collected separately.

\section{qPCR analysis of in vitro samples.}

DNA-free RNA ( $1 \mu \mathrm{g})$ was used as template for cDNA synthesis using the RevertAid First Strand cDNA Synthesis Kit (Thermo, Waltham, MA, U.S.A.) using random hexamer primers according to the manufacturer's instructions. In each experiment, a cDNA synthesis reaction mix, excluding the reverse-transcriptase enzyme but including RNA and all other reaction components, was prepared and used as a negative control. The synthesized cDNA was used as template in qPCR reactions with the Takara SYBR Premix Ex Taq II (TakaraBio, Shiga, Japan) according to the manufacturer's instructions. The negative control samples were included in each qPCR experiment to confirm the lack of any significant amount of genomic DNA contamination in the cDNA samples. Relative expression was normalized with $g y r A$ as an internal reference and Cmm382 in M9 medium was used as control. qPCR experiments were performed on a Stratagene Mx3000p instrument (Agilent Technologies, Santa Clara, CA, U.S.A.). 
Primers used for the qPCR experiments are listed in Supplementary Table S1. For data analysis, average threshold cycle values were calculated for each gene of interest on the basis of three independent biological samples, normalized, and used to calculate relative transcript levels as described (Pfaffl 2001).

\section{qPCR analysis of in planta samples.}

The procedure used for quantitative reverse-transcriptase PCR analysis was essentially as previously described (Chalupowicz et al. 2010). For each time point, two separated pools of three inoculated stem junction parts (1 cm long) were cut from tomato plants infected with $C m m 382, \Delta v a t r l$, and $\Delta v a t r 2$ and used for isolation of RNA with a MasterPure RNA Purification Kit (Epicentre Biotechnologies, Madison, WI, U.S.A.). RNA quantification was performed with a NanoDrop 1000 spectrophotometer (Thermo Fisher Scientific, Waltham, MA, U.S.A.). To remove residual genomic DNA, the isolated RNA was treated with DNase (Turbo DNA-free; Ambion Inc., Austin, TX, U.S.A.). Reverse transcription was carried out with $1 \mu \mathrm{g}$ of total RNA using a qScript cDNA synthesis kit (Quanta Biosciences, Gaithersburg, MD, U.S.A.). Triplicates of cDNA synthesis were performed for $45 \mathrm{~min}$ at $42^{\circ} \mathrm{C}$. In each experiment, a cDNA synthesis reaction mix, excluding the reversetranscriptase enzyme but including RNA and all other reaction components, was prepared and used to confirm the absence of DNA contamination in RNA samples. The resulting cDNA was subjected to PCR amplification with Power SYBR green PCR master mix (Applied Biosystems, Foster City, CA, U.S.A.). Real-time reaction was performed with a 7300 realtime PCR system (Applied Biosystems). Relative expression was normalized with gyrA and bipA as internal references and inoculated plants at time of inoculation were used as controls. Relative quantification and statistical analysis were performed with the data analysis program of real-time PCR software (Applied Biosystems).

\section{RNA-seq.}

cDNA libraries were synthesized using the TruSeq Sample Prep Kit V2 SetA (Illumina, San Diego, CA, U.S.A.) (Ref 15025063, no polyA) using $350 \mathrm{ng}$ of clean RNA from each sample (wild-type and mutants in LB and IMM) and were analyzed on a HighSeq 2000 instrument (Illumina) at Technion, Haifa, Israel. Resulting data were analyzed using the Cufflinks software package. The normalized abundance of a gene transcript was determined based on normalized reads per kilobase per million mapped reads. Differential expression of genes was determined using the Cuffdiff 2 algorithm (Trapnell et al. 2013) within Cufflinks, using the three biological replicates for each strain or condition as input. The following criteria were set for differential expression: between LB and IMM, a minimum of twofold change with $P$ value $<0.05$ to identify genes that are positively regulated by Vatr1 or Vatr2 during infection. i) Upregulation of the gene in Cmm382 in IMM compared with LB was at least twofold, with $P$ value $<0.05$. ii) Fold upregulation of the gene in the mutant in IMM compared with LB was at least twofold lower than the upregulation for Cmm382, under the same comparison. iii) The relative abundance of the gene in $C m m 382$ in IMM compared with the gene in the mutant in IMM was at least twofold higher with $P$ value $<0.05$.

To identify candidate genes that are negatively regulated by Vatr1 and Vatr2 during infection, the following criteria were set. i) Upregulation of the gene in the mutant in IMM compared with the gene in $C m m 382$ in IMM was at least twofold with $P$ value $<0.05$. ii) Fold upregulation of the gene in Cmm382 in IMM compared with LB was not statistically significant. Comparisons of read sequences between the various treatments are available on the Tel Aviv University Department of Molecular Biology and Ecology of Plants website.

\section{ACKNOWLEDGMENTS}

We thank O. Yaron from the Functional Genomics Lab at Tel Aviv University for cDNA library generation for RNA-seq experiments, D. Chalupowicz for ethylene measurements, and N. Kol for bioinformatics analysis of RNA-seq results. This research has received funding from the European Union's Seventh Framework Programme (FP7/2007-2013) under grant agreement number 239415 . This study was supported by the Israeli Ministry of Absorption and by the United States-Israel Binational Agricultural Research and Development Fund (grant number IS-4403-11C to S. Manulis-Sasson, I. Barash, and G. Sessa).

\section{LITERATURE CITED}

Balaji, V., Mayrose, M., Sherf, O., Jacob-Hirsch, J., Eichenlaub, R., Iraki, N., Manulis-Sasson, S., Rechavi, G., Barash, I., and Sessa, G. 2008. Tomato transcriptional changes in response to Clavibacter michiganensis subsp. michiganensis reveal a role for ethylene in disease development. Plant Physiol. 146:1797-1809.

Balaji, V., Sessa, G., and Smart, C.D. 2011. Silencing of host basal defense response-related gene expression increases susceptibility of Nicotiana benthamiana to Clavibacter michiganensis subsp. michiganensis. Phytopathology 101:349-357.

Bramucci, E., Milano, T., and Pascarella, S. 2011. Genomic distribution and heterogeneity of MocR-like transcriptional factors containing a domain belonging to the superfamily of the pyridoxal-5'-phosphate dependent enzymes of fold type I. Biochem. Biophys. Res. Commun. 415:88-93.

Chalupowicz, L., Cohen-Kandli, M., Dror, O., Eichenlaub, R., Gartemann, K.H., Sessa, G., Barash, I., and Manulis-Sasson, S. 2010. Sequential expression of bacterial virulence and plant defense genes during infection of tomato with Clavibacter michiganensis subsp. michiganensis. Phytopathology 100:252-261.

Choi, H. K., Park, N. Y., Kim, D. I., Chung, H. J., Ryu, S., and Choi, S. H 2002. Promoter analysis and regulatory characteristics of vvhBA encoding cytolytic hemolysin of Vibrio vulnificus. J. Biol. Chem. 277:4729247299.

Coaker, G. L., Willard, B., Kinter, M., Stockinger, E. J., and Francis, D M. 2004. Proteomic analysis of resistance mediated by Rcm 2.0 and Rcm 5.1, two loci controlling resistance to bacterial canker of tomato. Mol. Plant-Microbe Interact. 17:1019-1028.

Davis, M. J., Gillaspie, A. G., Jr., Vidaver, A. K., and Harris, R. W. 1984. Clavibacter: A new genus containing some phytopathogenic coryneform bacteria, including Clavibacter xyli subsp. xyli sp. nov., subsp. nov. and Clavibacter xyli subsp. cynodontis subsp. nov., pathogens that cause ratoon stunting disease of sugarcane and bermudagrass stunting disease. Int. J. Syst. Bacteriol. 34:107-117.

Dreier, J., Meletzus, D., and Eichenlaub, R. 1997. Characterization of the plasmid encoded virulence region pat-1 of phytopathogenic Clavibacter michiganensis subsp. michiganensis. Mol. Plant-Microbe Interact. 10:195-206.

Fatmi, M., Schaad, N. W., and Bolkan, H. A. 1991. Seed treatment for eradicating Clavibacter michiganensis subsp. michiganensis from naturally infected tomato seeds. Plant Dis. 75:383-385.

Gartemann, K. H., and Eichenlaub, R. 2001. Isolation and characterization of IS1409, an insertion element of 4-chlorobenzoate-degrading Arthrobacter sp. strain TM1, and development of a system for transposon mutagenesis. J. Bacteriol. 183:3729-3736.

Gartemann, K. H., Abt, B., Bekel, T., Burger, A., Engemann, J., Flugel, M., Gaigalat, L., Goesmann, A., Grafen, I., Kalinowski, J., Kaup, O., Kirchner, O., Krause, L., Linke, B., McHardy, A., Meyer, F., Pohle, S., Ruckert, C., Schneiker, S., Zellermann, E. M., Puhler, A., Eichenlaub, R., Kaiser, O., and Bartels, D. 2008. The genome sequence of the tomato-pathogenic actinomycete Clavibacter michiganensis subsp. michiganensis NCPPB382 reveals a large island involved in pathogenicity. J. Bacteriol. 190:2138-2149.

Jahr, H., Dreier, J., Meletzus, D., Bahro, R., and Eichenlaub, R. 2000. The endo-beta-1,4-glucanase CelA of Clavibacter michiganensis subsp. michiganensis is a pathogenicity determinant required for induction of bacterial wilt of tomato. Mol. Plant-Microbe Interact. 13:703-714.

Kirchner, O., Gartemann, K. H., Zellermann, E. M., Eichenlaub, R., and Burger, A. 2001. A highly efficient transposon mutagenesis system for the tomato pathogen Clavibacter michiganensis subsp. michiganensis. Mol. Plant-Microbe Interact. 14:1312-1318.

Kovacikova, G., and Skorupski, K. 2002. Regulation of virulence gene 
expression in Vibrio cholerae by quorum sensing: HapR functions at the aphA promoter. Mol. Microbiol. 46:1135-1147.

Laine, M. J., Nakhei, H., Dreier, J., Lehtila, K., Meletzus, D., Eichenlaub, R., and Metzler, M. C. 1996. Stable transformation of the gram-positive phytopathogenic bacterium Clavibacter michiganensis subsp. sepedonicus with several cloning vectors. Appl. Environ. Microbiol. 62:1500-1506.

Linhart, C., Halperin, Y., and Shamir, R. 2008. Transcription factor and microRNA motif discovery: The Amadeus platform and a compendium of metazoan target sets. Genome Res. 18:1180-1189.

Meletzus, D., and Eichenlaub, R. 1991. Transformation of the phytopathogenic bacterium Clavibacter michiganense subsp. michiganense by electroporation and development of a cloning vector. J. Bacteriol. 173:184-190.

Meletzus, D., Bermphol, A., Dreier, J., and Eichenlaub, R. 1993. Evidence for plasmid-encoded virulence factors in the phytopathogenic bacterium Clavibacter michiganensis subsp. michiganensis NCPPB382. J. Bacteriol. 175:2131-2136.

Pedley, K. F., and Martin, G. B. 2003. Molecular basis of Pto-mediated resistance to bacterial speck disease in tomato. Annu. Rev. Phytopathol. 41:215-243.

Perry, R. D., and Fetherston, J. D. 2011. Yersiniabactin iron uptake: Mechanisms and role in Yersinia pestis pathogenesis. Microbes Infect. 13:808-817.

Pfaffl, M. W. 2001. A new mathematical model for relative quantification in real-time RT-PCR. Nucleic Acids Res. 29:e45.

Ramos, J. L., Martinez-Bueno, M., Molina-Henares, A. J., Teran, W., Watanabe, K., Zhang, X., Gallegos, M. T., Brennan, R., and Tobes, R. 2005. The TetR family of transcriptional repressors. Microbiol. Mol. Biol. Rev. 69:326-356.

Rigali, S., Derouaux, A., Giannotta, F., and Dusart, J. 2002. Subdivision of the helix-turn-helix GntR family of bacterial regulators in the FadR, HutC, MocR, and YtrA subfamilies. J. Biol. Chem. 277:12507-12515.

Rivas, R., Vizcano, N., Buey, R. M., Mateos, P. F., Martinez-Molina, E., and Velazquez, E. 2001. An effective, rapid and simple method for total
RNA extraction from bacteria and yeast. J. Microbiol. Methods 47:59-63. Savidor, A., Teper, D., Gartemann, K. H., Eichenlaub, R., Chalupowicz, L., Manulis-Sasson, S., Barash, I., Tews, H., Mayer, K., Giannone, R. J., Hettich, R. L., and Sessa, G. 2012. The Clavibacter michiganensis subsp. michiganensis -tomato interactome reveals the perception of pathogen by the host and suggests mechanisms of infection. J. Proteome Res. 11:736-750.

Shao, C. P., and Hor, L. I. 2001. Regulation of metalloprotease gene expression in Vibrio vulnificus by a Vibrio harveyi LuxR homologue. J. Bacteriol. 183:1369-1375.

Stork, I., Gartemann, K. H., Burger, A., and Eichenlaub, R. 2008. A family of serine proteases of Clavibacter michiganensis subsp. michiganensis: chpC plays a role in colonization of the host plant tomato. Mol. Plant Pathol. 9:599-608.

Tauch, A., Zheng, Z., Puhler, A., and Kalinowski, J. 1998. Corynebacterium striatum chloramphenicol resistance transposon Tn5564: Genetic organization and transposition in Corynebacterium glutamicum. Plasmid 40:126-139.

Trapnell, C., Hendrickson, D.G., Sauvageau, M., Goff, L., Rinn, J. L., and Pachter, L. 2013. Differential analysis of gene regulation at transcript resolution with RNA-seq. Nat. Biotechnol. 31:46-53.

Tsiantos, J. 1987. Transmission of bacterium Corynebacterium michiganense pv. michiganense by seeds. J. Phytopathol. 119:142-146.

\section{AUTHOR-RECOMMENDED INTERNET RESOURCES}

BlastP database: www.ncbi.nlm.nih.gov /Blast.cgi

ClustalX database: www.clustal.org

Cufflinks software: cufflinks.cbcb.umd.edu

ImageJ software: rsbweb.nih.gov/ij

Pfam database: pfam.sanger.ac.uk

Tel Aviv University Department of Molecular Biology and Ecology of Plants:

www.tau.ac.il/lifesci/departments/plant_s/members/sessa/sessa.html 